\title{
Energy-aware predictive control for electrified bus networks
}

\author{
Balázs Varga ${ }^{\mathrm{a}, *}$, Tamás Tettamantia ${ }^{\mathrm{a}}$, Balázs Kulcsár ${ }^{\mathrm{b}}$ \\ ${ }^{a}$ Department of Control for Transportation and Vehicle Systems, Budapest University of Technology and \\ Economics, Stoczek J. u. 2, H-1111, Budapest, Hungary \\ ${ }^{b}$ Department of Electrical Engineering, Chalmers University of Technology, Hörsalsvägen 9-11, SE-412-96, \\ Gothenburg, Sweden
}

\begin{abstract}
For an urban bus network to operate efficiently, conflicting objectives have to be considered: providing sufficient service quality while keeping energy consumption low. The paper focuses on energy efficient operation of bus lines, where bus stops are densely placed, and buses operate frequently with possibility of bunching. The proposed decentralized, bus fleet control solution aims to combine four conflicting goals incorporated into a multi-objective, nonlinear cost function. The multi-objective optimization is solved under a receding horizon model predictive framework. The four conflicting objectives are as follows. One is ensuring periodicity of headways by watching leading and following vehicles i.e. eliminating bus bunching. Equal headways are only a necessary condition for keeping a static, predefined, periodic timetable. The second objective is timetable tracking. A conflicting objective to the former is minimizing passenger waiting time. When more than the expected passengers are waiting for the bus it is desirable to haste the vehicle in order to prevent bunching. The final objective is energy efficiency. To this end, an energy consumption model is formulated considering battery electric vehicles with recuperation during braking. Different weighting strategies are compared and evaluated through realistic scenarios, realized in a validated microscopic traffic simulation environment. Simulation results suggest $3-8 \%$ network level energy saving compared to bus holding control while maintaining punctuality and periodicity of buses.
\end{abstract}

Keywords: Receding control; Multiobjective optimization; Bus bunching; Energy consumption; Passenger wait

\section{Introduction}

On busy urban arterials, especially during peak hours delay of public transport is critical. Due to the stochastic nature of traffic networks, adherence to a bus schedule is not guaranteed. The fluctuation of passenger demand, intersection delays, changing traffic conditions and different driving styles of bus drivers will lead to varying bus headways. Subsequently, due to the volatile nature of the system, any disparities in bus headways tend to increase over time, eventually resulting in bus bunching [1. Due to bunching the periodicity of arrivals fail and homogeneous service cannot be maintained [2]. Bus bunching has a well-established literature and several authors proposed different methods to overcome its adverse effect. 3. studied the effect of passenger arrival patterns on bunching, concluding that unexpected passenger demands and

\footnotetext{
${ }^{*}$ Corresponding author

Email addresses: varga.balazs@mail.bme.hu (Balázs Varga), tettamanti@mail.bme.hu (Tamás Tettamanti), kulcsar@chalmers.se (Balázs Kulcsár)
} 
traffic disturbance are the root causes of bunching. Due to bunching the periodicity of arrivals fail and homogeneous service cannot be kept 2. In 4 and 5 algorithms are developed to control the headway of consecutive buses. Bus bunching was mitigated with bus holding control in [6] [7] proposed a cooperative control algorithm for buses to balance headways. 8] provided a self-controlling algorithm to improve headway reliability without timetable. The above works focus solely on headway keeping, disregarding adhering to the schedule.

In addition to bunching, timetable reliability and passenger waiting times are two factors influencing service quality. At frequent lines, if schedule cannot be held and a bus arrives at the stop late or with a large headway gap, the number of passengers is winding up. It leads to non-homogeneous utilization of buses and therefore degradation of service quality. [9] provides a predictive method based on GPS position and timetable data. A common method in improving timetable reliability is via yielding priority to buses at signalized intersections [10]. In [10], a velocity control method considering bus-to-bus communication and green time extension is applied. Public transport reliability is addressed in [11] with bus holding and stop skipping strategies to minimize passenger waiting time.

On top of service quality, an emerging trend in public transport is the reduction of its environmental footprint, dependency on fossil fuels and carbon emissions. Several cities where pollution is a strong concern are shifting public transportation towards electrified vehicles [12. Electric vehicles have no tailpipe emissions, are quieter, more energy efficient and simpler, requiring less maintenance too. The need for increasing energy efficiency and the advances in driver assistance systems brought eco-cruise control strategies to life [13, [14, [15], 16]. In urban areas interaction with traffic control devices dictate energy savings [17. Speed advisory systems incorporating V2I communication with traffic lights can result in significant energy reduction [18. [19] gave different control strategies for different driving styles for automated electric vehicles, bearing energy efficiency in mind. In addition to energy management between stops, charging station allocation and charging strategies are in the spotlight 20, 21, 22. Instead of focusing on individual vehicles, urban transportation can be viewed as an independent component of a smart city power network [23. In a comprehensive literature survey, 223] examined different distributed electrified transport networks and suggested cooperative energy management strategies. 24] studied the feasibility of an electric bus network focusing on battery capacity, charging and impact on the grid. They concluded, it is necessary to consider an electric bus network as a whole, rather than looking at individual bus trips. The state of the art and potential challenges for vehicle to grid (V2G) technology were summarized in [25]. Sustainable V2G technology requires energy management [25], 26].

As seen from the literature review, operating a bus network efficiently has several aspects. Previous works focus on one or two objectives: either bus bunching or energy efficiency, but not in a combined way. Multi-objective, passenger demand-driven public transport receives increasing interest recently. [27] developed a bi-objective optimization model with the consideration of energy consumption and passenger waiting time in metro systems for energy efficiency. In [28, optimal control algorithms were considered, taking into account both headway and timetable keeping. 29] used predictive algorithms to improve public transport reliability. 30. proposed model predictive control (MPC) strategies to deal with both bunching and timetable adherence. We can then conclude, optimizing electrified bus networks can be approached from different directions based on the authors' intention (e.g. energy minimization, ensuring service homogeneity). Our approach considers all of the listed objectives.

Finding a compromise solution between energy consumption and service quality leads to a multi-objective optimization problem. Our approach to remedy bus bunching is solely based on velocity control. Velocity control received criticism in comparison to holding (e.g. [5]), due to drivers adherence to the predefined velocity or propagating delays to other participants of traffic. 
One of the main arguments for velocity control is that with the emergence of highly automated and autonomous vehicles accurate control can be achieved. In addition, the uncertain nature of traffic (intersection delays, congestion or slower vehicles ahead) can be ruled out with high penetration of autonomous vehicles communicating with each other and the infrastructure [31. In a highly automated environment, the sole remaining uncertainty is the behavior of humans, i.e. the passenger demand. The authors believe velocity control can be combined with holding strategies and can be adapted to the instantaneous state of the traffic network (i.e. quick change in control inputs).

The paper aims at providing novel control strategies for bus network operation, fit into one scalable, decentralized model predictive control (MPC) scheme. The goal is merging four conflicting, public bus service related objectives: timetable adherence, headway keeping, energy efficiency, and passenger demand. The main idea is to use short time horizon predictions and optimize the trajectory of every bus in real-time. The suggested rolling horizon policy is an adequate control solution to predict future obstacles along the route and incorporate reference trajectories from various sources. The control method focuses on network bunching, but in a way distributed, overlapped: every vehicle runs its own velocity controller and then they communicate their predicted trajectories among each other. The contribution of the paper is threefold:

- A cooperative speed advisory system is developed for a fleet of electrified buses.

- The algorithm is accounting for model based energy consumption minimization. Hence, the duality of public transport service quality and energy efficiency is addressed. The bunching routine is augmented with a first principal energy consumption model. This enables considering transport service quality in an energy efficient way.

- The elaborated algorithm is experimentally validated, considering different weighting strategies.

The remainder of this paper is organized as follows. Section 2 describes the cost function used for the optimization through four subsections: Section 2.1 introduces the timetable and headway tracking objectives and the formulation of their respective cost functions. In Section 2.2 a physical-based energy consumption model is proposed and transformed into the model predictive scheme. Section 2.3 introduces the method for penalizing passenger waiting time in the cost function. As conclusion of Section 2, Section 2.4 summarizes the objective function. Next, in Section 3 the simulation setup is introduced for comparative analysis of the formulated control method. In Section 4 simulation results are analyzed considering different weighting strategies. Finally, Section 5 concludes the findings of this paper. The structure of the paper is summarized in Figure 1 .

\section{Formulating the multi-objective cost function}

Buses operate on a route based on their timetable. When the schedule is tight, their trajectory shall be carefully planned while considering the schedule, passenger demand, and energy efficiency.

The proposed bus velocity control algorithm (referred hereafter as control algorithm) creates an optimal trajectory within a predefined prediction horizon, considering the schedule, the number of passengers waiting and the location of other buses. The trade-off in the optimization is the total energy consumed, which is modeled as a physical energy consumption model. During operation, due to irregular dwell times and traffic disturbances, they tend to get out of sync with the schedule and start bunching. The goal of the control algorithm is to ensure timetable and 

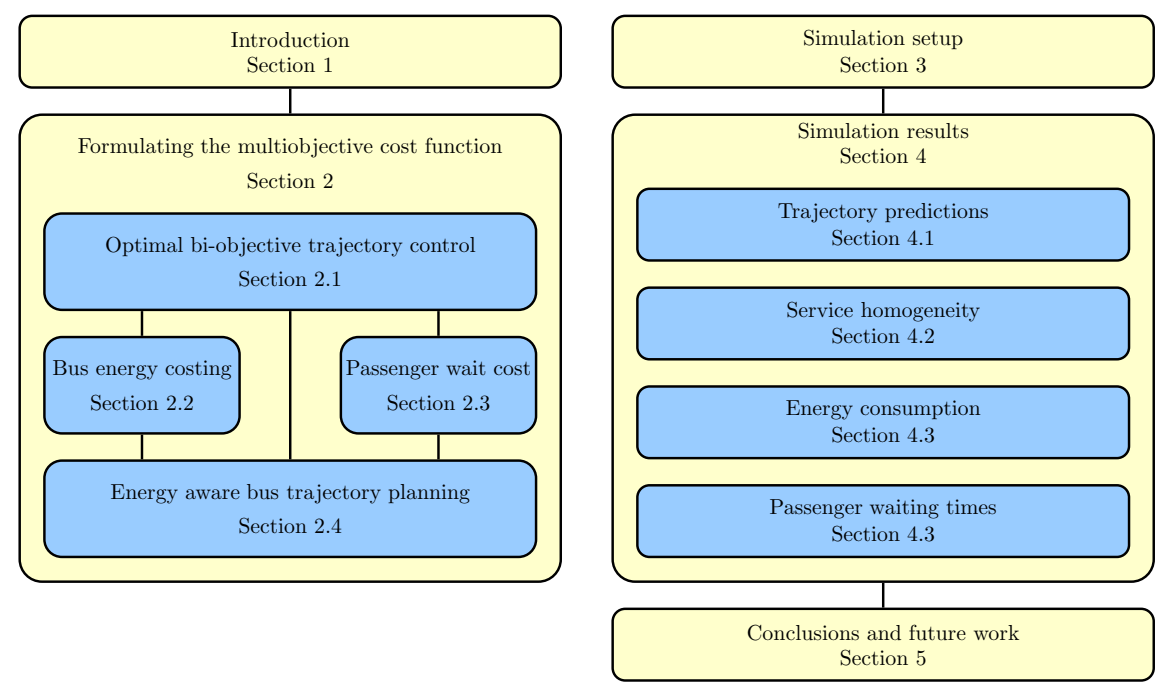

Figure 1: Paper layout

headway homogeneity in an energy efficient way while considering the number of passengers waiting at the bus stops. The controller calculates an optimal velocity profile $v_{\text {des }}$, obeying constraints set by the timetable and headway while minimizing the energy consumed.

To this end, three models are formulated. First, the longitudinal movement of a bus describing operations on a line is derived, in order to formulate reference trajectories which shall be adhered. This work considers three reference trajectories. For timetable adherence, an ideal desired trajectory $x_{t t}(t)$ is given. For providing equidistant headways between buses, the trajectory of the leading and following buses $x_{f w d}$ and $x_{b w d}$ respectively, are considered too. Second, an energy consumption model, based on the longitudinal velocity $v$ and acceleration of the vehicle is introduced, considering regenerative braking. The number of passengers $P_{o}$ on board the bus is also considered. This model is then fit into a model predictive framework, resulting in a more realistic approach for penalizing control input, albeit turning the cost function into a non-smooth one. Finally, passenger waiting times at bus stops are modeled. The passenger waiting model requires the number of bus stops ahead $j, \ldots, Y$ and the actual number of passengers $P_{j}, \ldots, P_{Y}$ as well as their time spent waiting at each stop $T_{j}, \ldots, T_{Y}$. The layout and interconnection of the proposed subsystems are presented in Figure 2 .

\subsection{Optimal bi-objective trajectory control}

Movement along the route is characterized by a longitudinal car following model. The discrete-time model for the bus dynamics (position $x(k)$, velocity $v(k)$ and acceleration $a(k)$ ) can be given as follows [32]:

$$
\begin{array}{r}
x(k+1)=x(k)+v(k) \Delta t, \\
v(k+1)=v(k)+a(k) \Delta t, \\
a(k)=\frac{1}{\tau}\left(v_{\text {des }}(k)-v(k)-v_{\text {dist }}(k)\right)
\end{array}
$$

where position $x(k+1)$ and velocity $v(k+1)$ denote the states over the time period of $[k \Delta t,(k+$ 1) $\Delta t$ ] with discrete time step index $k$ and sampling time $\Delta t . v_{\text {des }}(k)$ is the desired velocity at time step $k . \tau$ is a model parameter capturing the sensitivity of drivers to the change of 


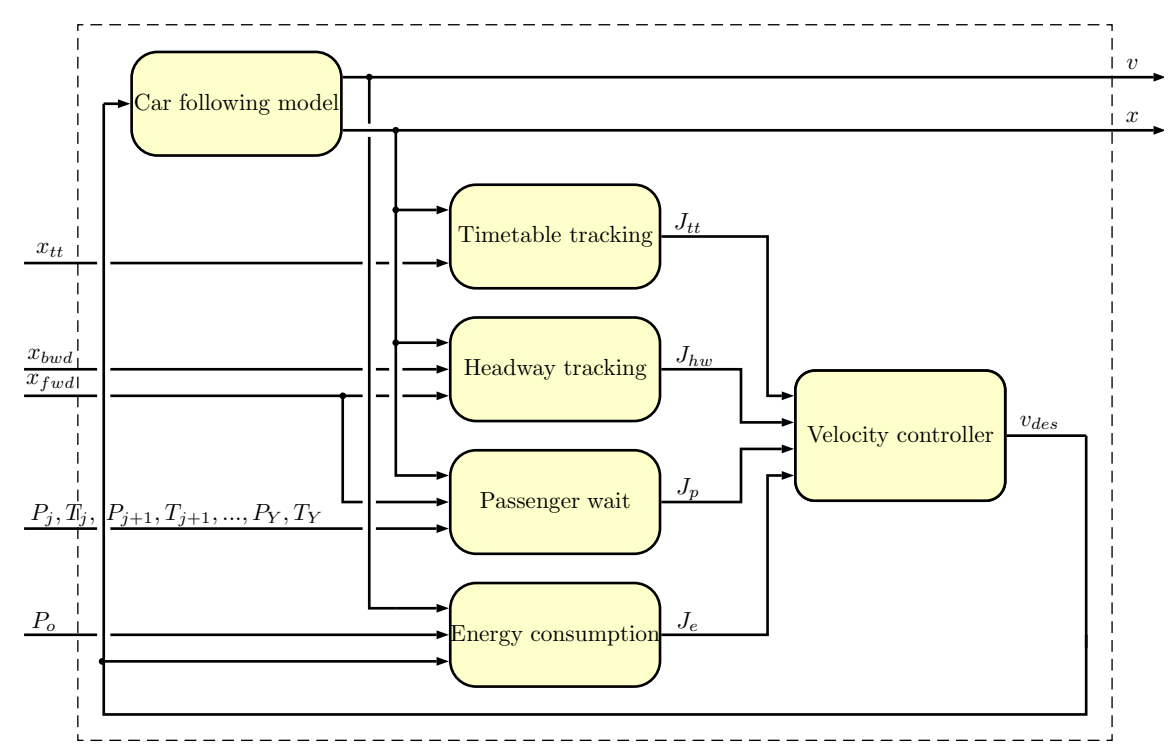

Figure 2: Layout of the proposed control system architecture

their desired velocity. According to 33 it shall be calibrated between $1.25 \mathrm{~s}$ and $2.5 \mathrm{~s}$. Too small values would result in rapid acceleration or deceleration towards the desired velocity. With autonomous vehicles these parameters could change, but still it is preferred to mimic the behavior of human drivers so their presence does not perturb traffic significantly and does not disturb other drivers participating in traffic [34. In addition, an additive error structure is proposed to include the adverse effect of other vehicles participating in traffic: $v_{\text {dist }}(k)=$ $\beta\left(v_{d e s}(k)-v_{m a c}(k)\right)$, with $v_{m a c}(k)$ being the macroscopic velocity on the link the bus travels on. $\beta \in[0,1]$ describes relaxation of bus speed towards a traffic dependent equilibrium velocity. With this term, road link specific obstacles such as traffic lights or bottlenecks can be considered. The smaller $\beta$ is the slower vehicles adjust their velocity to the macroscopic velocity 35, 36.

The above equations can be written into state space form with $v_{d e s}(k)$ being the controlled variable of the system: it serves as a display to the driver or a strict reference in case of autonomous driving. $X(k)=[v(k), x(k)]^{T}$ is the vector of system states at time step $k$. Finally, $v_{m a c}(k)$ is the traffic disturbance. The state space representation of the system is therefore:

$$
\left[\begin{array}{l}
v(k+1) \\
x(k+1)
\end{array}\right]=\left[\begin{array}{cc}
1-\frac{\Delta t}{\tau} & 0 \\
\Delta t & 1
\end{array}\right]\left[\begin{array}{l}
v(k) \\
x(k)
\end{array}\right]+\left[\begin{array}{c}
\frac{\Delta t}{\tau}(1-\beta) \\
0
\end{array}\right] v_{\text {des }}(k)+\left[\begin{array}{l}
\beta \\
0
\end{array}\right] v_{m a c}(k) .
$$

To be able to handle the first two control objectives (i.e. headway homogeneity and timetable adherence) the proposed longitudinal bus dynamic model is augmented with three error terms $z_{1}, z_{2}$ and $z_{3} . z_{1}(k)=x_{\text {des }}(k)-x(k)$ is the difference between an idealized trajectory for adhering the timetable $x_{d e s}(k)$ and the actual position of the bus. The second error term $z_{2}(k)=x_{f w d}(k)-x(k)$ denotes the forward-looking headway tracking (i.e. bunching in relation to the bus ahead). $z_{2}(k)$ is the difference between the actual position of the controlled vehicle and the shifted position of the leading bus. If the actual headway between the two buses is larger than the prediction horizon, the reference trajectory $x_{r e f}(k)$ is known for every time iteration. (The leading bus has already traveled on this trajectory so this information exists). Otherwise, the trajectory prediction of the leading bus is also considered, see Figure 5. Finally, $z_{3}(k)=x_{b w d}(k)+x(k)$ is the backward-looking headway error. The position of the following bus 
$x_{b w d}(k)$ is known for the current time instant $k$, however unknown for any future $k+i, i=1,2, \ldots$ time steps. However, in the distributed control scheme it can be assumed, that the following vehicle is also running the same predictive algorithm. The predicted trajectory of the following bus can be communicated to the controlled one and can be used for prediction. The predicted trajectory of the following bus is also shifted by one time headway, see Figure 3 .

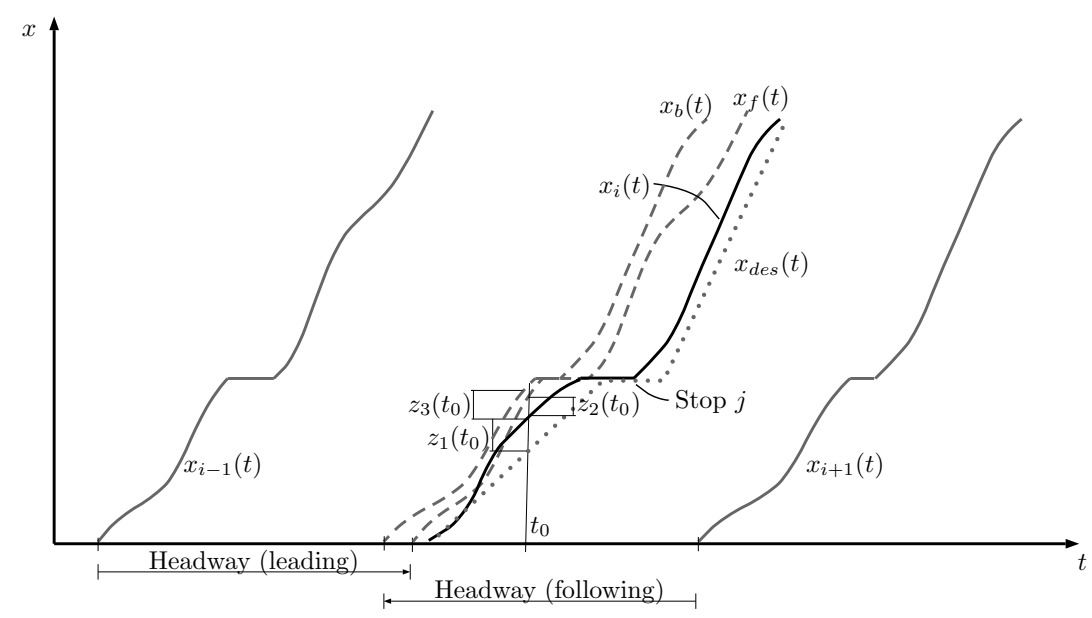

Figure 3: Reference trajectories for timetable tracking $x_{r e f}$, Forward bunching minimization $x_{f w d}$, Backward bunching minimization $x_{b w d}$

The above three reference trajectories (two objectives: timetable tracking and headway tracking) will serve as performance outputs which shall be minimized in an optimal way for each individual bus with the control action. In matrix form, using the system states $X(k)$ :

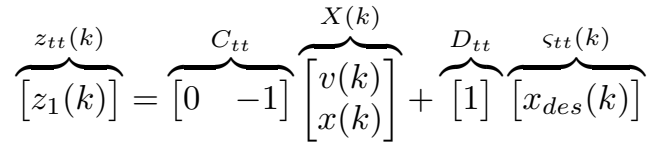

$$
\begin{aligned}
& \overbrace{\left[\begin{array}{l}
z_{2}(k) \\
z_{3}(k)
\end{array}\right]}^{z_{h w}(k)}=\overbrace{\left[\begin{array}{ll}
0 & -1 \\
0 & -1
\end{array}\right]}^{C_{h w}} \overbrace{\left[\begin{array}{l}
v(k) \\
x(k)
\end{array}\right]}^{X(k)}+\overbrace{\left[\begin{array}{ll}
1 & 0 \\
0 & 1
\end{array}\right]}^{D_{h w}} \overbrace{\left[\begin{array}{l}
x_{f w d}(k) \\
x_{b w d}(k)
\end{array}\right]}^{\operatorname{shw}(k)}
\end{aligned}
$$

The control-oriented model is used as the basis of a shrinking horizon MPC design [37. The goal of the controller is calculating an optimal velocity profile along its route in an energy efficient way. The shrinking horizon MPC prediction length depends on the scheduled travel time to the next bus stop. The interval between the actual $t_{0}$ and the desired arrival time to the next stop $t_{E T A}$ is split into $N$ equidistant time samples, see Figure 4. In every time-step, the prediction horizon decreases by one. By the last time step, the bus shall arrive at the desired stop. To avoid small or even negative horizon lengths (due to lateness or being close to the stop) the horizon length is bounded by a lower bound $N_{\min }=5$.

$$
N=\min \left\{N_{\min }, \frac{t_{E T A}-t_{0}}{\Delta t}\right\} .
$$




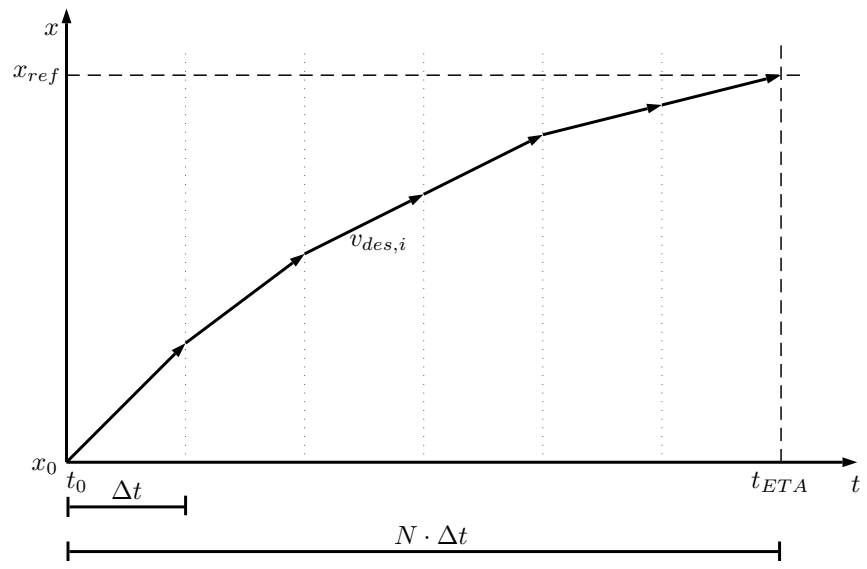

Figure 4: MPC horizon length calculation

Opposed to [5], in the model predictive scheme, the backward headway has little use. When the time headway between the controlled bus and its follower is greater than the prediction length, no data can be used from that vehicle. In Figure 5 the triangles represent the prediction horizon. The encircled sections of the leading and following bus trajectories are used as the reference trajectories. It can be seen, that only a fraction of the following bus's trajectory prediction is useful. In addition, the forward reference trajectory also consists of some of the prediction steps of the leading bus.

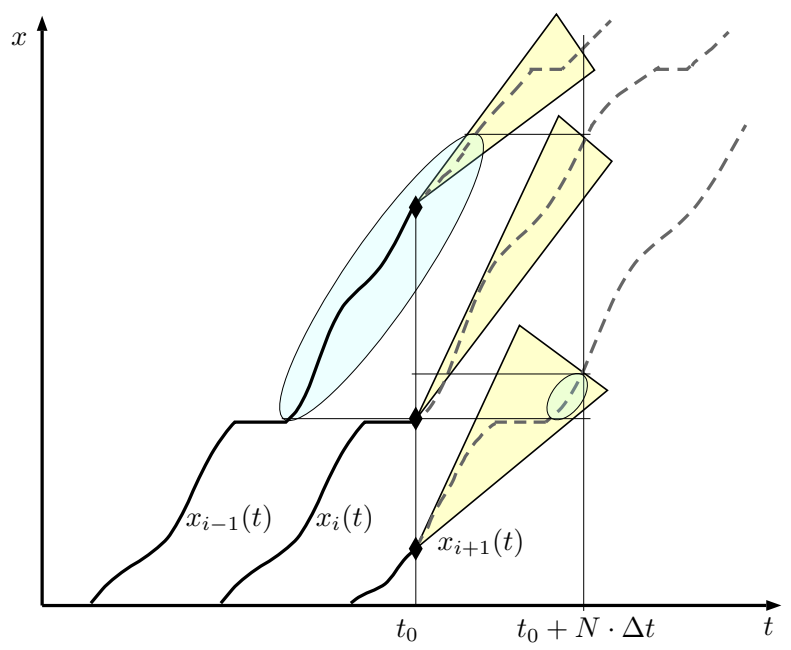

Figure 5: Trajectory predictions: The controlled vehicle $x_{i}$ at $t_{0}$ considers the leading and following vehicles' actual and predicted trajectories.

Consider the state space representation in Eq. (4) and tracking performances Eq. (5) and Eq. (6) and extend it for $N$ horizon, see Eq. (8). The system state $X(k)$ is measured at time step $k$. Then, for a finite horizon length $N$ the future states $X(k+i \mid k)$ are calculated along with the corresponding control inputs $u(k+i-1 \mid k)$ and the external reference signals $\varsigma_{t t}(k+i-1 \mid k)$ and $\varsigma_{h w}(k+i-1 \mid k)$. Predicted state is denoted as $X(k+i \mid k)$, where time step $k$ at the right side within the parentheses denotes the current time, and $k$ at the left side the prediction step 
with running index $i=1,2, \ldots, N$. The same notation applies for the control input the external signals and the performance outputs $z_{t t}(k+i \mid k)$ or $z_{h w}(k+i \mid k)$. The sampling time of the model is $\Delta t=1 \mathrm{~s}$.

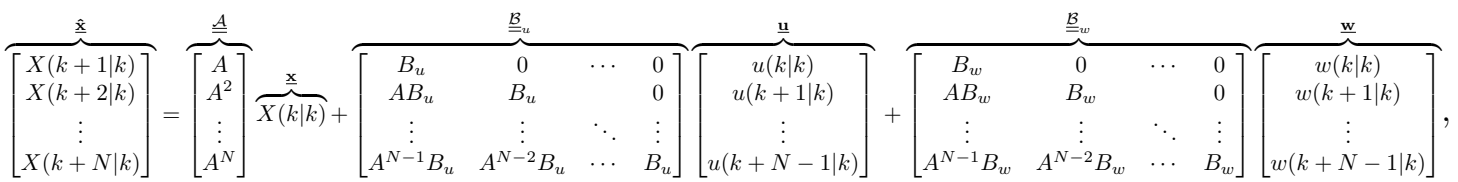

$\overbrace{\left[\begin{array}{c}z_{t t}(k+1 \mid k) \\ z_{t t}(k+2 \mid k) \\ \vdots \\ z_{t t}(k+N \mid k)\end{array}\right]}^{\hat{\underline{z}}_{t t}}=\overbrace{\left[\begin{array}{cccc}C_{t t} & 0 & \cdots & 0 \\ 0 & C_{t t} & & 0 \\ \vdots & \vdots & \ddots & \vdots \\ 0 & 0 & \cdots & C_{t t}\end{array}\right]}^{\underline{\mathcal{C}}_{t t}} \overbrace{\left[\begin{array}{c}X(k+1 \mid k) \\ X(k+2 \mid k) \\ \vdots \\ X(k+N \mid k)\end{array}\right]}^{\hat{\hat{\mathbf{x}}}}+\overbrace{\left[\begin{array}{cccc}D_{t t} & 0 & \cdots & 0 \\ 0 & D_{t t} & & 0 \\ \vdots & \vdots & \ddots & \vdots \\ 0 & 0 & \cdots & D_{t t}\end{array}\right]}^{\underline{\mathcal{D}}_{t t}} \overbrace{\left[\begin{array}{c}\varsigma_{t t}(k+1 \mid k) \\ \varsigma_{t t}(k+2 \mid k) \\ \vdots \\ \varsigma_{t t}(k+N \mid k)\end{array}\right]}^{\hat{\underline{S}}_{t t}}$,

\begin{tabular}{|c|c|c|c|c|c|c|c|c|c|}
\hline$\underline{\hat{\mathbf{z}}}_{h w}$ & \multicolumn{3}{|c|}{$\underline{\underline{\mathcal{C}}}_{h w}$} & $\underbrace{\underline{n}}_{\hat{\mathbf{x}}}$ & & \multicolumn{3}{|c|}{$\underline{\underline{\mathcal{D}}}_{h w}$} & $\underline{\hat{\underline{\xi}}}_{h w}$ \\
\hline$\overbrace{\left[z_{h w}(k+1 \mid k)\right]}$ & $\overparen{\Gamma C_{h u}}$ & 0 & 0 & $\overbrace{[X(k+1 \mid k)]}$ & & $\overparen{\left[D_{h u}\right.}$ & 0 & 0 & $\overbrace{\left[\varsigma_{h w}(k+1 \mid k)\right]}$ \\
\hline$z_{h w}(k+2 \mid k)$ & 0 & $C_{h w}$ & 0 & $X(k+2 \mid k)$ & & 0 & $D_{h w}$ & 0 & $\varsigma_{h w}(k+2 \mid k)$ \\
\hline$\left[\begin{array}{c}\vdots \\
z_{h w}(k+N \mid k)\end{array}\right]$ & 0 & $\begin{array}{l}\vdots \\
0\end{array}$ & $C_{h w}$ & {$\left[\begin{array}{c}\vdots \\
X(k+N \mid k)\end{array}\right]$} & 1 & $\dot{0}$ & $\begin{array}{l}\vdots \\
0\end{array}$ & $\begin{array}{c}\vdots \\
D_{h w}\end{array}$ & $\left\lfloor\varsigma_{h w}(k+N \mid k)\right]$ \\
\hline
\end{tabular}

Notations in Equation (8) are summarized below:

- $X(k)$ is the vector of state variables: $X(k)=[v(k), x(k)]^{T}$.

- A denotes the state matrix.

- $B_{u}$ is the control input matrix containing coefficients for the desired velocity: $B_{u}=$ $\left[\frac{\Delta t}{\tau}(1-\beta), 0\right]^{T}$.

- $u(k)$ is the controlled variable (decision variable). The only control input to the system is the desired velocity of the bus $u(k)=v_{\text {des }}(k)$.

- $B_{w}$ is a block diagonal disturbance matrix, consisting of $\left[\begin{array}{ll}\beta & 0\end{array}\right]^{T}$ matrices.

- $w(k)=v_{m a c}(k)$ is the distrubance vector.

- $\varsigma_{t t}(k)$ and $h_{h w}$ comprises the reference trajectories i.e. $\varsigma_{t t}(k)=x_{d e s}(k)$ and $\varsigma_{h w}(k)=$ $\left[x_{f w d}(k), x_{b w d}(k)\right]^{T}$.

- $z_{t t}(k)$ and $z_{h w}(k)$ are the vector of performance outputs $z_{t t}(k)=z_{1}(k), z_{h w}=\left[z_{2}(k) z_{3}(k)\right]^{T}$.

- $C_{t t}$ and $C_{h w}$ are the output matrices.

- $D_{t t}$ and $D_{h w}$ are direct feedthrough matrices of the reference trajectories.

The quadratic cost-functions for headway and timetable reference tracking can be constructed from with the help of $\underline{\underline{\mathbf{z}}}_{t t}, \underline{\underline{\mathbf{z}}}_{h w}$ and $\underline{\mathbf{u}}$ :

$$
J_{t t}(k)=\frac{1}{2}\left[\underline{\hat{\mathbf{z}}}_{t t}^{T} \underline{\underline{\mathcal{Q}}}_{t t} \hat{\mathbf{z}}_{t t}+\underline{\mathbf{u}}^{T} \underline{\underline{\mathcal{R}}}_{t} \underline{\underline{\mathbf{u}}}\right],
$$




$$
J_{h w}(k)=\frac{1}{2}\left[\underline{\hat{\mathbf{z}}}_{h w}^{T} \underline{\underline{\mathcal{Q}}}_{h w} \underline{\hat{\mathbf{z}}}_{h w}+\underline{\mathbf{u}}^{T} \underline{\underline{\mathcal{R}}}_{h w} \underline{\underline{\mathbf{u}}}\right] .
$$

$\underline{\hat{\mathbf{z}}}_{t t}, \underline{\hat{\mathbf{z}}}_{h w}$ and $\underline{\mathbf{u}}$ denote stacked vectors of the predicted referece trajectory errors and the control input (desired velocity) at every time iteration. With the two cost functions the two objectives of timetable- and headway tracking are separated. Introduce positive semidefinite weighting matrices $Q_{t t}=\operatorname{diag}\left(q_{z, 1}\right)$ and $Q_{h w}=\operatorname{diag}\left(q_{z, 2}, q_{z, 3}\right)$, where $q_{z, 1}, q_{z, 2}, q_{z, 3}$ are tuning parameters for their respective reference trajectories. Higher value on a weight means more emphasis on that tracking objective. $R_{t t}$ and $R_{h w}$ are scalar weights penalizing the control input $v_{\text {des }}(k)$. $Q_{t t}, Q_{h w}, R_{t t}$ and $R_{h w}$ weights are also extended for $N$ horizon as follows: $\underline{\underline{\mathcal{Q}}}_{t t}=\operatorname{diag}\left(Q_{t t, 1}, Q_{t t, 2}, \ldots, Q_{t t, N}\right), \underline{\underline{\mathcal{Q}}}_{h w}=\operatorname{diag}\left(Q_{h w, 1}, Q_{h w, 2}, \ldots, Q_{h w, N}\right)$, $\underline{\underline{\mathcal{R}}}_{t t}=\operatorname{diag}\left(R_{t t, 1}, R_{t t, 2}, \ldots, R_{t t, N}\right)$ and $\underline{\underline{\mathcal{R}}}_{h w}=\operatorname{diag}\left(R_{h w, 1}, R_{h w, 2}, \ldots, R_{h w, N}\right)$.

Note that $\underline{\hat{\mathbf{x}}}$ does not directly appear in the cost function. The first state is velocity and adding a weight to that would penalize the kinetic energy of the bus (i.e. demand small velocity). Kinetic energy is considered in this scheme via a more accurate energy consumption model. The state $x(k)$ is the absolute position of the bus. Minimizing absolute position would be a physically unreasonable choice.

By inserting Eq. (9) into Eq. (10) and Eq. (11) and following some simple algebraic steps, the cost functions simplify to:

$$
\begin{aligned}
& J_{t t}(k)=\frac{1}{2} \underline{\mathbf{u}}^{T}\left(\underline{\underline{\mathcal{B}}}_{u}^{T} \underline{\underline{\mathcal{C}}}^{T} \underline{\underline{\mathcal{Q}}}_{t t} \underline{\underline{\mathcal{C}}}_{\underline{\underline{\mathcal{B}}}}+\underline{\underline{\mathcal{R}}}_{t t}\right) \underline{\mathbf{u}}^{T} \\
& +\left(\underline{\mathbf{x}}^{T} \underline{\underline{\mathcal{A}}}^{T} \underline{\underline{\mathcal{C}}}^{T} \underline{\underline{\mathcal{Q}}}_{t t} \underline{\underline{\mathcal{C}}} \underline{\underline{\mathcal{B}}}_{u}+\underline{\mathbf{w}}^{T} \underline{\underline{\mathcal{B}}}_{w}^{T} \underline{\underline{\mathcal{C}}}^{T} \underline{\underline{\mathcal{Q}}}_{t t} \underline{\underline{\mathcal{C}}} \underline{\underline{\mathcal{B}}}_{u}+\underline{\hat{\zeta}}^{T} \underline{\underline{\mathcal{D}}}^{T} \underline{\underline{\mathcal{Q}}}_{t t} \underline{\underline{\mathcal{C}}} \underline{\underline{\mathcal{B}}}_{u}\right) \underline{\mathbf{u}} .
\end{aligned}
$$

and

$$
\begin{array}{r}
J_{h w}(k)=\frac{1}{2} \underline{\underline{\mathbf{u}}}^{T}\left(\underline{\underline{\mathcal{B}}}_{u}^{T} \underline{\underline{\mathcal{C}}}^{T} \underline{\underline{\mathcal{Q}}}_{h w} \underline{\underline{\mathcal{C}}}_{u}+\underline{\underline{\mathcal{R}}}_{h w}\right) \underline{\mathbf{u}} \\
+\left(\underline{\mathbf{x}}^{T} \underline{\underline{\mathcal{A}}}^{T} \underline{\underline{\mathcal{C}}}^{T} \underline{\underline{\mathcal{Q}}}_{h w} \underline{\underline{\mathcal{C}}} \underline{\underline{\mathcal{B}}}_{u}+\underline{\mathbf{w}}^{T} \underline{\underline{\mathcal{B}}}_{w}^{T} \underline{\underline{\mathcal{C}}}^{T} \underline{\underline{\mathcal{Q}}}_{h w} \underline{\underline{\mathcal{C}}} \underline{\underline{\mathcal{B}}}_{u}+\underline{\hat{\boldsymbol{\zeta}}}^{T} \underline{\underline{\mathcal{D}}}^{T} \underline{\underline{\mathcal{Q}}}_{h w} \underline{\underline{\mathcal{C}}} \underline{\underline{\mathcal{B}}}_{u}\right) \underline{\mathbf{u}} .
\end{array}
$$

subject to:

$$
v_{\min } \leq v_{\text {des }}(k) \leq v_{\max } .
$$

As a constraint for the optimization, it is assumed that the control input is limited: the lower limit $v_{\min }=0 \mathrm{~km} / \mathrm{h}$, since negative velocity is not allowed, $v_{\max }$ is constrained by the legal speed limit on the link (e.g. $v_{\max }=50 \mathrm{~km} / \mathrm{h}$ ). For detailed deduction steps, please refer to 30 .

In summary, this section proposed three reference trajectories for each bus, which shall be tracked based on a cost function. This approach enables considering timetable and headway adherence, however does not incorporate the cost of operating buses on a route directly.

\subsection{Bus energy costing}

In this section, an energy consumption model is proposed for electric public transport vehicles, based on their velocity profile. Further in this section, the proposed model is simplified and reformulated into a piecewise cost function. The model discusses the share of resistances and the effect of recuperation. The energy consumption model is based on the longitudinal motion of the vehicle (Section 2.1) and the losses occurring during operation. Energy consumption is obtained by summing the power required to move the vehicle in every time instant $k$. The modeling framework is based on the model used by [38] and 39].

The tractive power at the wheels $P_{w}$ consists of five terms: 


$$
P_{\text {roll }}(k)=\mu \cdot m \cdot g \cdot \cos \theta \cdot v(k)
$$

is the rolling resistance with $m$ the total vehicle mass and inertia, $g=9.81 \mathrm{~m} / \mathrm{s}^{2}$ the gravitational constant, $v(k)$ the velocity and $\mu$ the rolling resistance factor. Rolling resistance for buses on dry asphalt it is approximately 0.01. $\theta$ is the road inclination. Weight consists of the curb weight of the vehicle $m_{v e h}$ and the weight of passengers $m_{p}: m=m_{v e h}+P_{o} m_{p}$. The average weight of a passenger $m_{p}$ is assumed to be $80 \mathrm{~kg}$ and $P_{o}$ is the number of passengers on board the bus. The total vehicle mass is passenger count dependent, its value changes after each bus stop. Fortunately, passenger load is measurable, so there is no obstacle including it as a varying parameter

The extra power required when driving uphill (or downhill) is

$$
P_{g}(k)=m \cdot g \cdot \sin \theta \cdot v(k) .
$$

Next the air drag is analyzed.

$$
P_{\text {drag }}(k)=\frac{1}{2} \cdot c_{w} \cdot \rho \cdot A_{f} \cdot v(k)^{3}
$$

where $c_{w}$ the drag coefficient, $\rho=1.293 \mathrm{~kg} / \mathrm{m}^{3}$ the air density and $A_{f}$ the frontal area of the vehicle.

The last two terms are related to acceleration and deceleration $a(k)$ of the vehicle. For convenience, denote acceleration with $a^{+}(k)$ and deceleration with $a^{-}(k)$. The power required to accelerate is:

$$
P_{a c c}(k)=m \cdot v(k) \cdot a^{+}(k)
$$

This term is zero when decelerating, regeneration is taken into account in the following term:

$$
P_{\text {regen }}(k)=m \cdot v(k) \cdot a^{-}(k) \cdot \eta_{\text {regen }}
$$

where this term is negative, because it is not a loss, but a power gain. $\eta_{\text {regen }}$ stands for the efficiency of regeneration.

The power required to move the vehicle is the summation of the five terms in Equations (15)-(19):

$$
P_{w}(k)=P_{\text {roll }}(k)+P_{g}(k)+P_{\text {drag }}(k)+P_{a c c}(k)+P_{\text {regen }}(k) .
$$

Next, drivetrain of buses is examined from efficiency point of view. Figure 6 depicts the losses of an electric powertrain. The efficiency of batteries of electric vehicles varies in a wide range. For Lithium-ion batteries it is around $\eta_{\text {batt }}=90-95 \%$. The efficiency of the electric machine varies based on its operation point, but the aim is to keep it as high as possible. The power electronics (e.g. inverter, cabling, etc.) loss is between $1-4 \%$, thus $\eta_{p e}=96-99 \%$. The electric motor efficiency is assumed to be between $\eta_{m o t}=90-97 \%$. Finally, the mechanical efficiency of the powertrain is $\eta_{p t}=98 \%$. The regeneration for the buses is assumed to be $50 \%$ of the braking energy, which is fed back to the battery, however, it greatly varies based on the intensity of braking (i.e. using friction brakes, [40, [41]). Feeding back means the direction of the energy flow is reversed, the losses in the drivetrain will apply again. The losses arising during operation are summarized in the Sankey diagram in Figure6. 


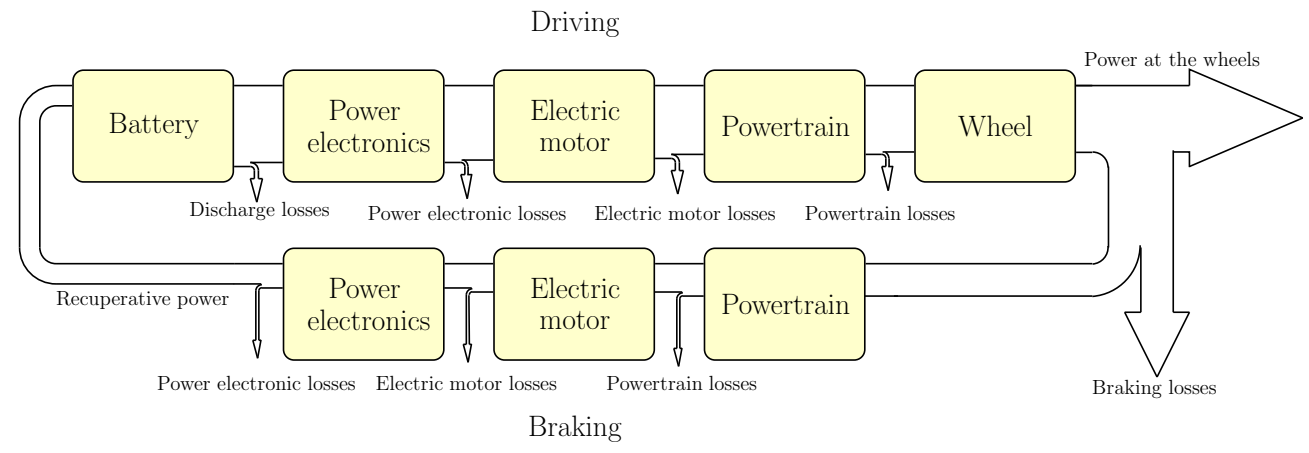

Figure 6: Sankey diagram of an electric powertrain with regenerative braking

The total energy consumption of a vehicle within the prediction horizon (between $k=1$ and $k=N)$ is calculated as:

$$
E_{\text {cons }}(k)=\sum_{k=1}^{N} \frac{P_{\text {roll }}(k)+P_{g}(k)+P_{\text {drag }}(k)+P_{a c c}(k)}{\eta_{\text {batt }} \cdot \eta_{p e} \cdot \eta_{\text {mot }} \cdot \eta_{p t}}+P_{\text {regen }}(k) \cdot \eta_{\text {batt }} \cdot \eta_{p e} \cdot \eta_{\text {mot }} \cdot \eta_{p t},
$$

assuming discrete time.

The next aim is to reformulate the energy consumption model, proposed in Section 2.2, in a way it can be fit into a cost function to penalize energy consumption of the vehicle along its route.

The power required to move the vehicle equals the energy consumption in one time step:

$$
P(k)=\frac{P_{\text {roll }}(k)+P_{\text {drag }}(k)+P_{g}(k)+P_{a c c}(k)}{\eta_{b a t t} \cdot \eta_{p e} \cdot \eta_{\text {mot }} \cdot \eta_{p t}}+P_{\text {regen }}(k) \cdot \eta_{b a t t} \cdot \eta_{p e} \cdot \eta_{\text {mot }} \cdot \eta_{p t} .
$$

In equation 222 the rolling resistance $P_{\text {roll }}(k)$, the resistance due to upgrade $P_{g}(k)$ and air drag $P_{\text {drag }}(k)$ are independent from the control input. In the quadratic minimization problem constants do not affect the result of the optimization, they just offset the cost, therefore they can be omitted. Furthermore, it makes the optimization much easier for two reasons: i) keeping terms with second or third order power of the velocity (state) would result in higher order polynomial as a cost function; ii) weighting the cost function is much easier when only the significant term (i.e. acceleration) is left. Weighting the total energy consumption would work towards slowing down the bus as lower velocity means lower energy consumption (neglecting auxiliary losses). On the other hand weighting acceleration directly penalizes rapid accelerations and decelerations which is the most significant portion of energy consumption.

The acceleration (and deceleration) $a(k)$ can be written based on the car following model (Section 2.1). Next, substitute Eq. (3) into Eq. (22) and group efficiencies into two parameters $\eta_{a c c}=\eta_{b a t t} \cdot \eta_{p e} \cdot \eta_{\text {mot }} \cdot \eta_{p t}$ and $\eta_{\text {reg }}=\eta_{b a t t} \cdot \eta_{p e} \cdot \eta_{\text {mot }} \cdot \eta_{p t} \cdot \eta_{\text {regen }}$ for $P_{a c c}(k)$ and $P_{\text {regen }}(k)$, respectively. The the equations become:

$$
\begin{gathered}
P_{a c c}(k)=-\frac{m \cdot v^{2}(k)}{\tau \cdot \eta_{a c c}}+\frac{m \cdot v(k)}{\tau \cdot \eta_{a c c}} \cdot v_{d e s}(k), \\
P_{\text {regen }}(k)=-\frac{\eta_{r e g} \cdot m \cdot v^{2}(k)}{\tau}+\frac{\eta_{r e g} \cdot m \cdot v(k)}{\tau} \cdot v_{\text {des }}(k) .
\end{gathered}
$$


In equations (23) and (24) the first two terms are also independent from $v_{d e s}(k)$. The last term is linearly dependent.

In the finite horizon optimization problem velocity of the bus $v(k)$ is contained in the predicted state vector $\underline{\hat{\mathbf{x}}}$. The control input dependent part of $\underline{P}_{a c c}^{\prime}(k)$ and $\underline{P}_{r e g}^{\prime}(k)$ for $N$ horizon are:

$$
\begin{aligned}
& \underline{P}_{a c c}^{\prime}(k)=\underline{\mathbf{u}}^{T} \cdot \frac{m}{\tau \cdot \eta_{a c c}} \cdot \underline{\underline{\mathcal{S}}} \cdot \underline{\hat{\mathbf{x}}}, \\
& \underline{P}_{r e g}^{\prime}(k)=\underline{\mathbf{u}}^{T} \cdot \frac{\eta_{r e g} \cdot m}{\tau} \cdot \underline{\underline{\mathcal{S}}} \cdot \underline{\hat{\mathbf{x}}},
\end{aligned}
$$

where $\mathcal{S}$ is a row selector matrix for the velocity $v(k)$ over the prediction horizon:

$$
\underline{v}(k)=\underline{\underline{\mathcal{S}}} \underline{\hat{\mathbf{x}}}=\left[\begin{array}{cccc}
S & 0 & \ldots & 0 \\
0 & S & & 0 \\
\vdots & \vdots & \ddots & \vdots \\
0 & 0 & \ldots & S
\end{array}\right]\left[\begin{array}{c}
X(k+1 \mid k) \\
X(k+2 \mid k) \\
\vdots \\
X(k+N \mid k)
\end{array}\right], \quad S=[1,0] .
$$

For the sake of simplicity, define $\underline{\underline{\kappa}}_{a c c}$ and $\underline{\underline{\kappa}}_{r e g}$ as the time independent coefficient of $\underline{\hat{\mathbf{x}}}$ in $\underline{P}_{a c c}^{\prime}(k)$ and $\underline{P}_{r e g}^{\prime}(k)$ are respectively:

$$
\begin{gathered}
\underline{\underline{\kappa}}_{a c c}=\frac{m}{\tau \cdot \eta_{a c c}} \cdot \underline{\underline{\mathcal{S}}}, \\
\underline{\underline{\kappa}}_{r e g}=\frac{\eta_{r e g} \cdot m}{\tau} \cdot \underline{\underline{\mathcal{S}}} .
\end{gathered}
$$

Furthermore, from Eq. (8) $\underline{\hat{\mathbf{x}}}$ is

$$
\underline{\hat{\mathbf{x}}}=\underline{\underline{\mathcal{A}}} \underline{\mathbf{x}}+\underline{\underline{\mathcal{B}}} \underline{\mathbf{u}} .
$$

Next, substitute Eq. (30) into Eq. 25) and Eq. 26) and organize the resulting equations by $\underline{\mathbf{u}}$ :

$$
\begin{aligned}
& \underline{P}_{a c c}^{\prime}(k)=\underline{\mathbf{u}}^{T} \underline{\underline{\kappa}}_{a c c} \underline{\underline{\mathcal{A}}} \underline{\mathbf{x}}+\underline{\mathbf{u}}^{T} \underline{\underline{\kappa}}_{a c c} \underline{\underline{\mathcal{B}}} \underline{\mathbf{u}}, \\
& \underline{P}_{r e g}^{\prime}(k)=\underline{\mathbf{u}}^{T} \underline{\underline{\kappa}}_{r e g} \underline{\underline{\mathcal{A}}} \underline{\mathbf{x}}+\underline{\mathbf{u}}^{T} \underline{\underline{\kappa}}_{r e g} \underline{\underline{\mathcal{B}}} \underline{\mathbf{u}} .
\end{aligned}
$$

The power to accelerate and the regenerated power are quadratic polynomials of the control input u. Similar to Eq. 10, the cost function is sought in a quadratic form. The results of Eq. (31) and Eq. 32 can be written as two separate quadratic cost functions: $J_{e}^{+}(k)$ and $J_{e}^{-}(k)$ for accelerating and regenerative braking respectively. In order to penalize rapid accelerations or decelerations four weighting parameters are introduced: $\underline{\underline{\mathcal{W}}}_{a c c}, \underline{\underline{\mathcal{W}}}_{r e g}, \underline{\mathcal{V}}_{a c c}$ and $\underline{\mathcal{V}}_{r e g} \cdot \underline{\mathcal{V}}_{a c c}^{1 \times 1}$ is the coefficient for the first order part and $\underline{\underline{\mathcal{W}}}_{a c c}^{N \times N}$ is the diagonal, positive semi-definite coefficient matrix for the quadratic part of the cost function. The same rationale can be applied to $\underline{\underline{\mathcal{W}}}_{\text {reg }}$ and $\underline{\mathcal{V}}_{\text {reg }}$. Since the regeneration $P_{\text {reg }}$ is an energy gain, it has negative sign and decreases the cost. With the two additional quadratic cost functions $J_{e}^{+}(k)$ and $J_{e}^{-}(k)$ the optimization results in a non-smooth problem.

$$
\begin{gathered}
J_{e}^{+}(k)=\frac{1}{2} \underline{\mathbf{u}}^{T} \underline{\underline{\kappa}}_{a c c} \underline{\underline{\mathcal{B}}} \underline{\underline{\mathcal{W}}}_{a c c} \underline{\underline{\mathbf{u}}}+\underline{\underline{\mathbf{x}}}^{T} \underline{\underline{\mathcal{A}}}^{T} \underline{\underline{\kappa}}_{a c c}^{T} \underline{\mathcal{V}}_{a c c} \underline{\mathbf{u}}, \\
J_{e}^{-}(k)=-\frac{1}{2} \underline{\underline{\mathbf{u}}}^{T} \underline{\underline{\kappa}}_{r e g} \underline{\underline{\mathcal{B}}}_{\underline{\underline{\mathcal{W}}}} \underline{\underline{\mathbf{u}}}_{r e g} \underline{\underline{\mathbf{u}}}-\underline{\underline{\mathbf{x}}}^{T} \underline{\underline{\mathcal{A}}}^{T} \underline{\underline{\kappa}}_{r e g}^{T} \underline{\mathcal{V}}_{r e g} \underline{\mathbf{u}} .
\end{gathered}
$$

Switching between objective functions in Eq. (33) and Eq. (34) is formulated mathematically as follows. A selection between the cost functions shall be made for every time iteration in the 
prediction horizon. Exploiting some features of the matrices a simple solution can be given. First, consider the quadratic part in Eq. (33) or Eq. (34). In the cost function $\underline{\mathcal{B}}$ is a lower triangular matrix, $\underline{\underline{\kappa}}_{a c c}$ and $\underline{\underline{\kappa}}_{r e g}$ are non-square matrices with entries only in at $[i, 2 i]$. Due to their special structure, the result of their multiplication will be a diagonal $N \times N$ matrix. In the linear part of the cost functions $\underline{\underline{\kappa}}_{a c c}$ and $\underline{\underline{\kappa}}_{r e g}$ act as row selector matrices, yielding two $1 \times N$ vectors. For the sake of simplicity the selection between $J_{e}^{+}(k)$ and $J_{e}^{-}(k)$ is depending on the acceleration or deceleration (the relation of $v(k)$ and $v_{\text {des }}$ ) at the $i^{\text {th }}$ iteration at time step $k$. The piecewise quadratic cost function can be written as follows:

$$
\min _{u}\left\{\begin{array}{cc}
J_{e}^{+}(k, N) & v(k+i \mid k)<v_{\text {des }}(k+i \mid k)-\nu \\
0 & v(k+i \mid k)=\left(v_{\text {des }}(k+i \mid k)-\nu \ldots v_{\text {des }}(k+i \mid k)+\nu\right), \quad \forall i \in[1 . . N], \\
J_{e}^{-}(k, N) & v(k+i \mid k)>v_{\text {des }}(k+i \mid k)+\nu
\end{array}\right.
$$

subject to:

$$
\begin{aligned}
a_{\min } \leq \frac{1}{\tau}\left(v_{\text {des }}(k)-v(k)\right) & \leq a_{\max } \\
v_{\min } \leq v_{\text {des }}(k) & \leq v_{\max } .
\end{aligned}
$$

The acceleration and deceleration are bounded by $a_{\min }$ and $a_{\max }$ in order to avoid physically infeasible values. Furthermore, for numerical reasons a sufficiently small threshold parameter $\nu$ is introduced. The addition of energy consumption enables considering the varying passenger load. Furthermore rapid accelerations can be penalized while considering energy regeneration.

\subsection{Passenger wait cost}

Another term in the cost function is related to the number of passengers waiting at stops costing the secondary delay (waiting at the stops) of passengers. In this framework the number of passengers is assumed to be known (via measurement [42] or estimation [43]). In this model, the arrival of passengers at a bus stop is assumed to follow Poisson distribution with constant arrival rate $\lambda_{j}$, where the subscript denotes the $j^{\text {th }}$ bus stop [4].

The number of passengers $P$ waiting at stop $j$ can be written as:

$$
P_{j}(k+1)=P_{j}(k)+\alpha_{j}(k) .
$$

where $\alpha$ denotes the number of passengers arrived at time step $k: E\left[\alpha_{j}(k)\right]=\lambda_{j} \Delta t$. The cumulated passenger waiting time can be expressed as:

$$
T_{j}(k+1)=T_{j}(k)+P_{j}(k) \Delta t .
$$

The two equations can be cast into state space form as a double integrator system, with the states being $T_{j}(k)$ and $P_{j}(k)$ for each stop $j$.

$$
\left[\begin{array}{c}
T_{j}(k+1) \\
P_{j}(k+1)
\end{array}\right]=\left[\begin{array}{cc}
1 & \Delta t \\
0 & 1
\end{array}\right]\left[\begin{array}{c}
T_{j}(k) \\
P_{j}(k)
\end{array}\right]+\left[\begin{array}{l}
0 \\
1
\end{array}\right] \alpha_{j}(k)
$$

In addition, passenger number and waiting time are set to zero when the bus arrives and passengers board it. To this end, Eq. 40 is augmented with an additional logical term $\Gamma_{j}(x(k))$, which is function of the location of the bus. When the bus arrives at stop $j\left(z_{1}(k) \approx 0\right)$, then $\Gamma_{j}(x(k), k)$ is set to 1 , resetting the integrators, otherwise 0 . In addition, a new reset state $r(k)$ 
is appended which subtracts (i.e. resets) the accumulated value of state $P_{j}(k)$ and $T_{j}(k)$ when $\Gamma_{j}(x(k), k)=1$. The augmented state space becomes:

$$
\overbrace{\left[\begin{array}{l}
T_{j}(k+1) \\
P_{j}(k+1) \\
r_{j}(k+1)
\end{array}\right]}^{\underline{\chi_{j}(k+1)}}=\overbrace{\left[\begin{array}{ccc}
1 & \Delta t & -1 \\
0 & 1 & -1 \\
0 & 0 & 0
\end{array}\right]}^{\underline{\Lambda}} \overbrace{\left[\begin{array}{l}
T_{j}(k) \\
P_{j}(k) \\
r_{j}(k)
\end{array}\right]}^{\underline{\chi}_{j}(k)}+\overbrace{\left[\begin{array}{l}
0 \\
1 \\
0
\end{array}\right]}^{\underline{\beta}} \alpha_{j}(k)+\overbrace{\left[\begin{array}{l}
0 \\
0 \\
1
\end{array}\right]}^{\underline{\underline{\varepsilon}}} \Gamma_{j}(x(k), k) .
$$

In order to formulate an adequate cost function from the preliminary model, it shall be extended for $N$ horizon length:

$$
\begin{aligned}
& {\left[\begin{array}{c}
\underline{\chi}_{j}(k+1 \mid k) \\
\underline{\chi}_{j}(k+2 \mid k) \\
\vdots \\
\underline{\chi}_{j}(k+N \mid k)
\end{array}\right]=\left[\begin{array}{c}
\underline{\underline{\Lambda}} \\
\underline{\underline{\Lambda}}^{2} \\
\vdots \\
\underline{\underline{\Lambda}}^{N}
\end{array}\right] \underline{\underline{\chi}}_{j}(k)+\left[\begin{array}{cccc}
\underline{\underline{\beta}} & 0 & \cdots & 0 \\
\underline{\underline{\underline{\Lambda}}} \underline{\underline{\beta}} & \underline{\underline{\beta}} & & 0 \\
\vdots & \vdots & \ddots & \vdots \\
\underline{\underline{\Lambda}}^{N-1} \underline{\underline{\beta}} & \underline{\underline{\Lambda}}^{N-2} \underline{\underline{\beta}} & \cdots & \underline{\beta}
\end{array}\right]\left[\begin{array}{c}
\alpha_{j}(k \mid k) \\
\alpha_{j}(k+1 \mid k) \\
\vdots \\
\alpha_{j}(k+N-1 \mid k)
\end{array}\right]+} \\
& {\left[\begin{array}{cccc}
\underline{\underline{\mathcal{E}}} & 0 & \cdots & 0 \\
\underline{\underline{\underline{\mathcal{E}}}} & \underline{\underline{\mathcal{E}}} & & 0 \\
\vdots & \vdots & \ddots & \vdots \\
\underline{\underline{\Lambda}}^{N-1} \underline{\underline{\mathcal{E}}} & \underline{\underline{\Lambda}}^{N-2} \underline{\underline{\mathcal{E}}} & \cdots & \underline{\underline{\mathcal{E}}}
\end{array}\right]\left[\begin{array}{c}
\Gamma_{j}(k \mid k) \\
\Gamma_{j}(k+1 \mid k) \\
\vdots \\
\Gamma_{j}(k+N-1 \mid k)
\end{array}\right] }
\end{aligned}
$$

The cost function shall consider every bus stop $j=1 . . Y$, where $Y$ is the first stop after the leading bus $i-1$ (see Figure 7). Even though the rolling horizon MPC targets only the

\begin{tabular}{|c|c|c|}
\hline Bus $_{i} \stackrel{v_{i}}{\longrightarrow}$ & & Bus $s_{i-1} \stackrel{v_{i-1}}{\longrightarrow}$ \\
\hline Stop $_{1}$ & Stop $_{j}$ & StopY \\
\hline
\end{tabular}
next stop, the accumulation of waiting passengers at downstream stops are considered over the prediction horizon. Thus the cost function will be a sum of each predictive model:

Figure 7: Relevant bus stops for the passenger waiting time model (Stop . .Stop $\left._{Y}\right)$

$$
J_{p}(k)=\sum_{j=1}^{Y} \underline{\Omega}_{j}(k) .
$$

$\underline{\mathcal{X}}_{j}(k)$ is constructed from the predicted state vector as follows: $\underline{\mathcal{X}}_{j}(k)=\left[T_{j}(k \mid k), T_{j}(k+1 \mid k), \ldots T_{j}(k+N \mid k)\right]$. Thus, it is the passenger waiting time over the prediction horizon at stop $j$ at time step $k$. $\Omega$ is a weighting vector of appropriate size for the passenger waiting times.

\subsection{Energy aware bus trajectory planning}

The resulting cost function is the summation of the four cost function terms and constraints introduced in Section 2.1 2.3.

$$
\min _{v_{\text {des }}(k) . . v_{\text {des }}(k+N)} J(k)=J_{t t}(k)+J_{h w}(k)+J_{e}(k)+J_{p}(k),
$$

subject to:

$$
\begin{aligned}
a_{\min } \leq \frac{1}{\tau}\left(v_{\text {des }}(k)-v(k)\right) & \leq a_{\max } \\
v_{\min } \leq v_{\text {des }}(k) & \leq v_{\max } .
\end{aligned}
$$


The cost function in 44 creates a relationship between the four conflicting objectives. The free parameter in the optimization is the desired velocity of the bus over the prediction horizon. Initially, weighting scalars and matrices are chosen in such a way that each cost has a similar magnitude. By alternative selection of the weighting parameters one can formulate different control strategies (e.g. preferring objective $J_{e}$ over $J_{p}$ ). Alternatively, with proper weighting, public transport delay can be minimized in an energy-optimal way.

Since $J_{e}(k)$ and $J_{p}(k)$ are not linear cost functions (i.e. they depend on integer parameters), the resulting cost function will also be nonlinear. $J_{e}(k)$ is piecewise due to the separation of acceleration and deceleration. Furthermore, the passenger waiting model introduced a reset state $\Gamma_{j}(x(k), k)$, which is depending on a state variable $x(k)$. Due to the multiple decisions, the problem is non-smooth. However, when these decisions are eliminated the problem turns into a quadratic optimization which is analytically convenient. Therefore, we use sequential quadratic programming (SQP) 45. It is an iterative procedure that boils down the nonlinearity into repetitive sequence of quadratic approximations by QP. The optimization was solved with Matlab's fmincon function with the sqp algorithm. Considering all four cost functions and prediction horizon $N<50$, the problem can be solved just under the selected step time length $\Delta t=1 s$ of the model.

\section{Simulation setup}

In this section, the modeled bus line is introduced. For modeling purposes, a high-fidelity traffic simulator, VISSIM, is used [46]. The simulator can be used to generate different traffic scenarios and evaluate the developed control algorithm.

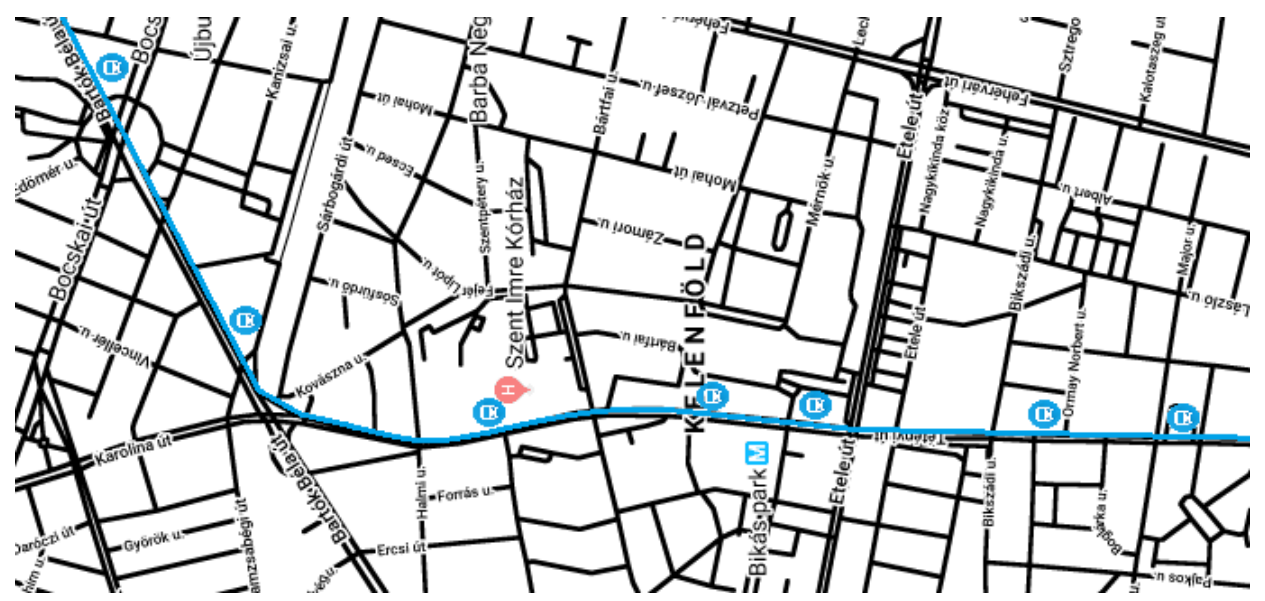

Figure 8: Modeled real-world section (source: Google maps, GPS coordinates: 47.465 N, 19.034 E)

A busy arterial in Budapest's $X I^{\text {th }}$ district serves as the basis of the analysis (Figure 8 . A $3 \mathrm{~km}$ long section of trunk bus line 7 is modeled, including 7 stops. In this area there is no dedicated lane, the bus travels in a mixed traffic environment along the route but there are two lanes so it can be overtaken. It is assumed that buses have priority at signalized intersections and the legal speed limit is $50 \mathrm{~km} / \mathrm{h}$. The time headway of the buses is $3 \mathrm{~min}$. Table 1 presents the passenger demand at each stop and the scheduled departure times (with entry to the network being 0$)$. 
Table 1: Bus arrival times (with entry to the network being 0 , in seconds) and passenger demand at each stop (passengers/hour)

\begin{tabular}{llll} 
Stop ID & Location $(m)$ & $\begin{array}{l}\text { Scheduled } \\
\text { arrival }(s)\end{array}$ & $\begin{array}{l}\text { Boarding volume } \\
(\text { pass } / h)\end{array}$ \\
\hline Stop 1 & 174 & 25 & 50 \\
Stop 2 & 402 & 75 & 50 \\
Stop 3 & 829 & 160 & 300 \\
Stop 4 & 1065 & 200 & 50 \\
Stop 5 & 1265 & 240 & 50 \\
Stop 6 & 1887 & 325 & 300 \\
Stop 7 & 2474 & 415 & 100 \\
\hline
\end{tabular}

\section{Simulation results}

In this section the effect of setting various costs on the control objectives is analyzed through the simulation environment. Initially, six weighting strategies are introduced (Figure 9):

a) Timetable tracking with $J_{t t}$ being the only considered cost.

b) Headway tracking, where only $J_{h w}$ is taken into account.

c) Balanced, where headway and timetable tracking are equally important $J=J_{t t}+J_{h w}$.

d) Passenger demand driven: on frequent lanes passengers usually do not consult the timetable [44. In order to avoid bunching (causing increased waiting times, [3) and minimize passenger waiting time. $J=J_{h w}+J_{p}$.

e) Cheap service driven. From the service providers' perspective minimizing energy consumption of their fleet is crucial as it has direct impact on their expenses. In addition, running buses based on a periodic timetable is the simplest in terms of planning. $J=J_{t t}+J_{e}$.

f) Finally, a balanced strategy is given, taking into account all four objectives: $J=J_{t t}+$ $J_{h w}+0.5 J_{e}+0.5 J_{p}$.

The analysis focuses on four metrics: shape of the predicted trajectories from a deterministic initial condition, service homogeneity, energy consumption (individual and network level) and finally passenger waiting times.

\subsection{Trajectory predictions}

Figure 10 depicts the predicted bus trajectories from one fixed point $(t=212 s, k=3)$ for each proposed control strategy. In this scenario the headway is $60 \mathrm{~s}$ and the prediction horizon is $100 \mathrm{~s}$. This means $40 \mathrm{~s}$ (varying based on the actual headway) of the predicted trajectory of the follower bus can be used. According to the timetable reference $x_{t t}$, the buses (the leader and the controlled) are running late.

In the following, the analysis focuses on strategies (d), (e), (f), as strategies similar to (a), (b), (c) were thoroughly analyzed in 30 . The first strategy is timetable tracking (Figure 10 (a)). Here, the only objective is following the trajectory predefined by an ideal schedule $x_{t t}$, which is done accurately. Next, headway tracking strategy is shown in Figure 10 (b). Since the forward-looking trajectory $x_{f w d}$ is more reliable than the backward-looking $x_{b w d}$ it is weighted $2: 1$. The predicted trajectory lies between the beforehand mentioned two references, closer to $x_{f w d}$. It is important to mention that the stops are not obeyed in the prediction as it is not 

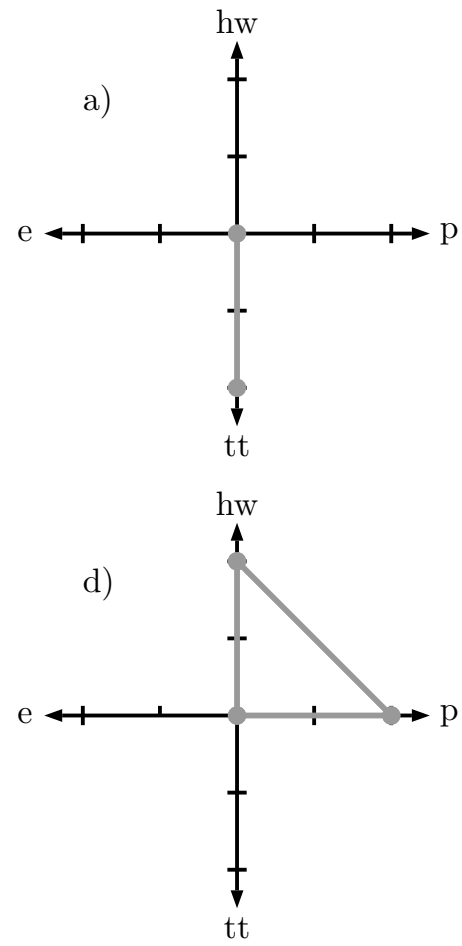
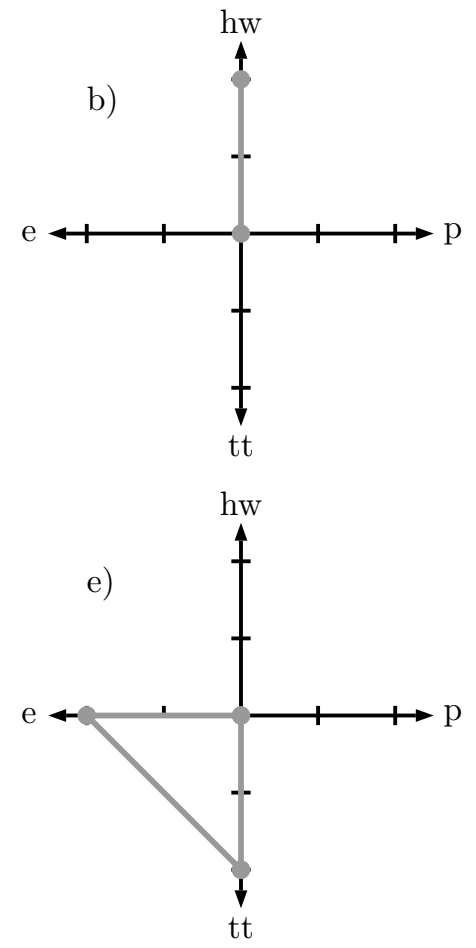
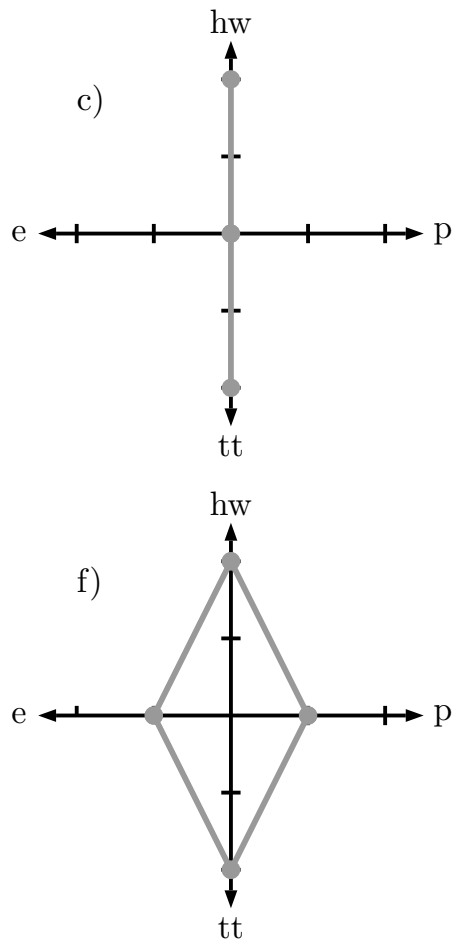

Figure 9: Proposed weighting strategies. a) Timetable tracking only, b) Headway tracking only, c) Balanced timetable and headway tracking, $d$ ) Passenger demand driven - headway tracking and waiting time minimization, e) Cheap service driven - timetable tracking and energy consumption minimization, $f$ ) Balanced, advanced timetable and headway tracking plus energy consumption and waiting time minimization. Abbrevations at each direction match the subscript of the respective cost function element

present in the constraints. It is not a problem since only the first step of the prediction is used as control input, the rest are discarded. In addition to that, the shape of the reference trajectories implies the presence of a stop and the desired velocity $v_{d e s}$ is chosen accordingly. The desired velocity is overwritten by the simulator and held at a stop while the passenger exchange is simulated, stop skipping does not happen as a result of the control. Next, the balanced strategy is shown in Figure 10 (c). In this case, both timetable and headway objectives are considered. In the passenger demand-driven scenario headway homogeneity and passenger waiting times are considered. Compared to the headway tracking scenario (Figure 10 (d)), when adding cost to passenger waiting times, vehicles will accelerate from the headway-defined trajectory as the passengers at a stop is accumulating. When energy consumption is penalized in the timetable tracking strategy, as shown in Figure 10 (e), accelerations and decelerations will become slower, causing minor delays. Finally, all four objectives are considered, however, passenger waiting time and energy consumption with $50 \%$ weight (Figure 10 (f)). Results are similar to the balanced (c) strategy, only with slower acceleration profiles. In addition, the predicted trajectory is knurled because regenerative braking is exploited. In a complete trajectory realization this effect is not visible. These trajectory predictions are for only one point in the space-time diagram, however, the aforementioned conclusions hold true for whole bus trajectories too. Using different weighting strategies, the value of each cost function changes based on its significance. The cost function values in the optimization shown in Figure 10 are summarized in Table 2 . Cost values increase as more conflicting objectives are considered. 

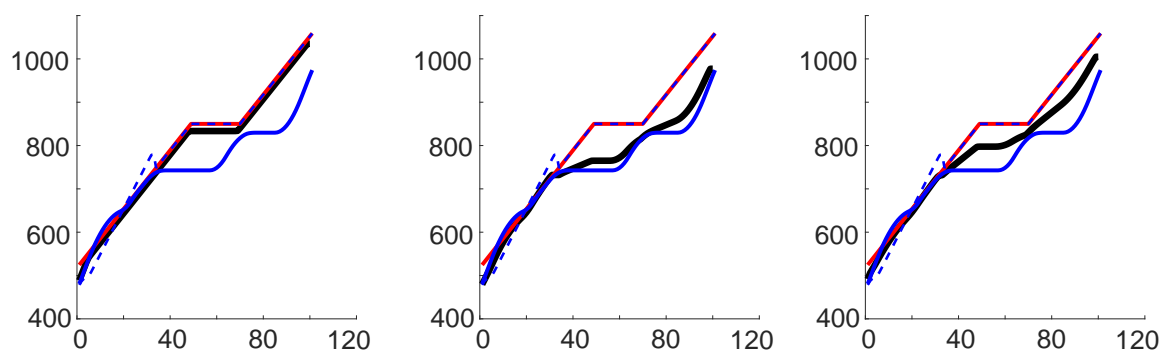

(a) Timetable tracking trajectory prediction

(b) Headway tracking trajec-

(c) Balanced trajectory pretory prediction diction
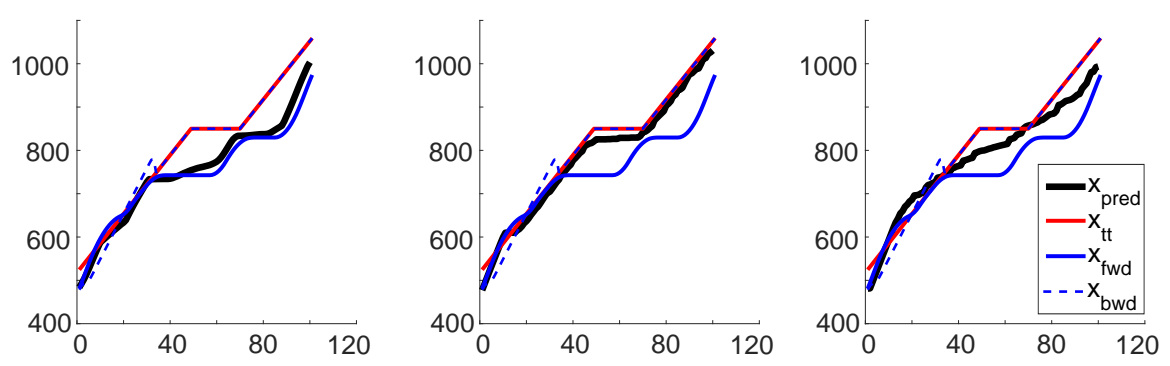

(d) Passenger demand driven prediction

(e) Cheap service driven pre- (f) Advanced balanced tradiction jectory prediction

Figure 10: Trajectory predictions from a fixed initial state with different weighting strategies compared to the reference trajectories (vertical axis: Position (m), horizontal axis: Time (s)) 
Table 2: Cost function values with different weighting strategies

\begin{tabular}{lllllll} 
& a) & b) & c) & d) & e) & f) \\
\hline$J_{t t}$ & 16 & 0 & 175 & 0 & 717 & 877 \\
\hline$J_{h w}$ & 0 & 149 & 167 & 166 & 0 & 879 \\
\hline$J_{e}$ & 0 & 0 & 0 & 0 & 430 & 473 \\
\hline$J_{p}$ & 0 & 0 & 0 & 268 & 0 & 129 \\
\hline
\end{tabular}

\subsection{Service homogeneity}

Next, the advanced balanced control strategy (f), (Figure 11) is compared to a benchmark scenario with bus holding control strategy (HCS) (simplified version of the HCS, proposed in 6]) over a $30 \mathrm{~min}$ long simulation with 3 min headways. With holding control buses always maintain $50 \mathrm{~km} / \mathrm{h}$ as their desired speed and depart from a stop based on a static timetable.

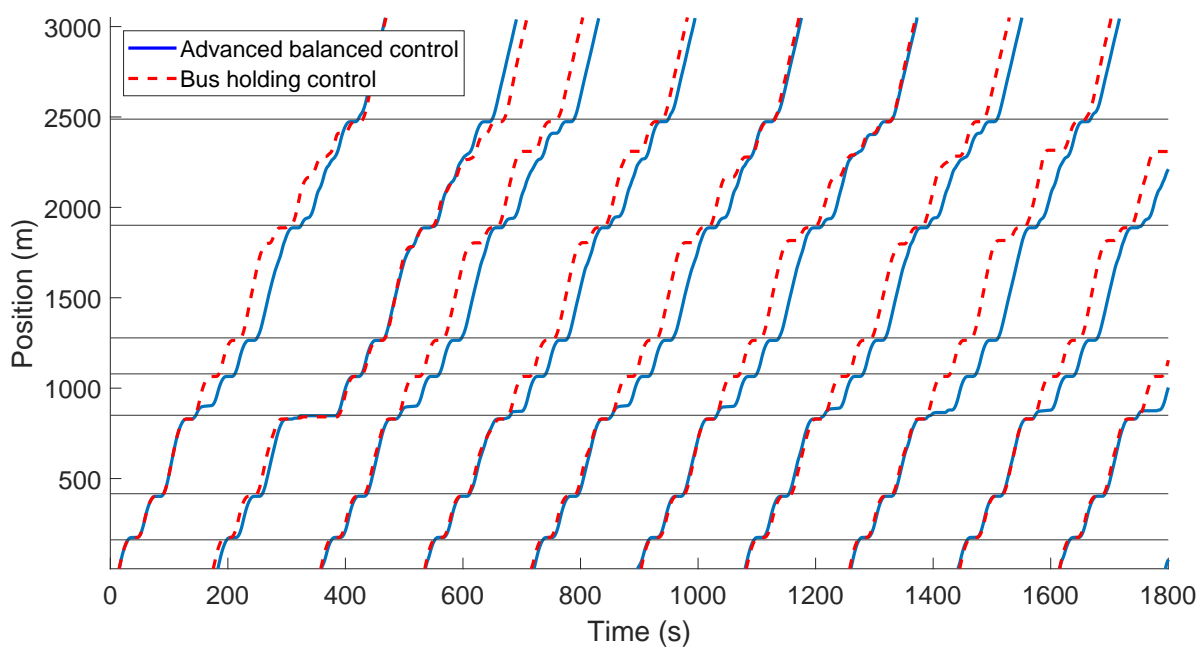

Figure 11: Bus trajectories over a 30 min simulation

For comparison, six locations are chosen and headways are compared statistically at those points. Note that, for the statistical results more trajectories are used than shown in Figure 11. In Table 3 the mean, standard deviation and the Kullback-Liebler (KL) divergence [4] are given. The KL divergence is a metric describing the 'distance' between two distributions. In this case, the distribution of headways is compared to the ideal headway distribution which is a uniform distribution with $180 \mathrm{~s}$ mean value and 0 variance.

The results are similar to the HCS scenario or slightly worse. It is because the controller considers multiple objectives that might conflict with headway homogeneity while in the holding scenario the buses only try to go as fast as possible between two stops. The mean values are similar in both cases, close to the ideal headway of $180 \mathrm{~s}(3 \mathrm{~min})$. Standard deviations and the KL divergence are smaller in the holding case compared to the controlled one, which means headways are more uniformly distributed. Headway homogeneity is only one metric to analyze the performance of a bus network. In the followings, energy efficiency is considered. 
Table 3: Statistics of the trajectories

\begin{tabular}{llll}
\hline \multicolumn{4}{c}{ Holding control } \\
Location $(\mathrm{m})$ & Mean $(\mathrm{s})$ & Variance $(\mathrm{s})$ & KL divergence \\
\hline 500 & 178.22 & 4.764 & 0.0003 \\
1000 & 179.44 & 21.732 & 0.0068 \\
1500 & 179.33 & 21.645 & 0.0067 \\
2000 & 178.67 & 21.389 & 0.0066 \\
2500 & 178.78 & 26.846 & 0.0099 \\
3000 & 178.56 & 24.053 & 0.0080 \\
\multicolumn{5}{l}{} \\
\hline \multicolumn{5}{l}{ Location (m) } & Mean (s) & Variance (s) & KL divergence \\
\hline 500 & 179.78 & 2.326 & 0.0001 \\
1000 & 182.00 & 22.425 & 0.0105 \\
1500 & 181.89 & 21.036 & 0.0109 \\
2000 & 181.56 & 23.805 & 0.0169 \\
2500 & 181.89 & 27.816 & 0.0132 \\
3000 & 182.00 & 28.068 & 0.0134
\end{tabular}

\subsection{Energy consumption}

The next metric is energy consumption. First, one bus is selected and its individual energy consumption is analyzed with different control strategies. In Figure 12 four bus trajectories are compared: holding control, PI control [48, energy-aware cheap control (d), and advanced balanced velocity control (f). The two benchmark control strategies (HCS and PI control) perform similarly in this metric as none of them considers energy consumption. The energy consumption is the lowest in the cheap control strategy. The balanced velocity control has smaller average velocity (spends more time in the network) thus it has lower energy consumption compared to HCS. If energy cost is not taken into account the decelerations are steeper while in the energy consumption aware scenarios with regenerative braking is considered, velocity profiles become smoother with a coasting phase before each stop. The average energy consumption on the modeled route is summarized in Figure 13 . The average energy consumption is 9.5\% smaller in the cheap velocity control and $4.3 \%$ smaller in the advanced balanced strategy compared to the holding strategy. The standard deviations of energy consumption (Figure 13 ) are similar to each other.

By arbitrarily selecting one vehicle does not say much about the total energy consumption of a public transport line. One bus may have good energy efficiency while it hinders following buses resulting in poorer energy efficiency for the follower. To this end, the energy consumption of individual buses is summed in the simulation scenario in Section 4.2 for three different control strategies (Figure 14). Simulation results suggest that the total energy consumption in the long run is as expected: the greedy HCS and the PI controller consume the most energy. The holding control tries to always reach the highest permitted velocity on the link, while the PI controller only considers headway deviations. On the other hand, the energy-aware cheap control (d), and advanced balanced velocity control (f) have better energy efficiency. Using the holding control as a benchmark (100\%), the energy-aware strategy consumes $8 \%$ less, while the advanced balanced velocity control consumes $3 \%$ less, considering a $3 \mathrm{~km}$ public transport line for 30 minutes. The PI controlled buses consume 3\% more compared to the HCS. Note that between $500-1100 \mathrm{~s}$ the total energy consumption of the balanced strategy is the lowest. Longer simulations can smoothen out such deviations. 


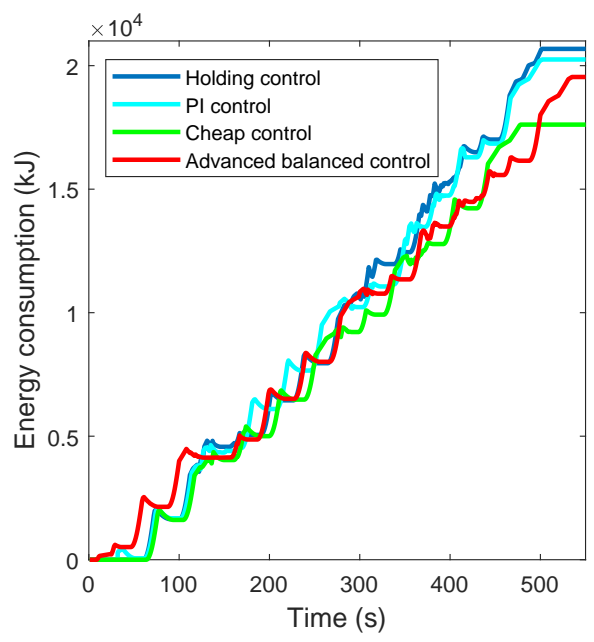

Figure 12: Energy consumption profile of one ve- Figure 13: Average energy consumption and regenhicle

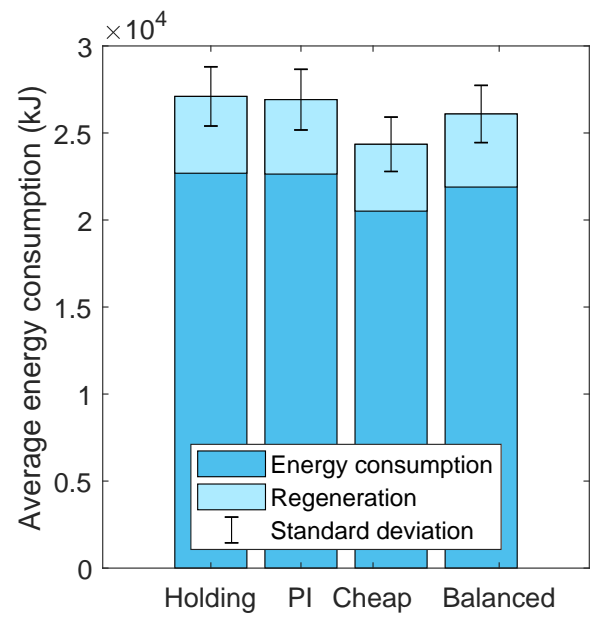

eration

\subsection{Passenger waiting times}

Finally, the effect of considering passenger waiting times is analyzed. Again, three scenarios are considered: the benchmark holding control, passenger driven (e) and the advanced balanced (f) strategy. In terms of total (cumulated) passenger waiting times at each stop, the balanced control performs worst (Figure 15). The passenger driven strategy performs slightly better than the HCS. The holding control strategy performs well in this metric because buses spend less time between stops and more time waiting at each stop so passengers who just arrived can board the bus instantly. The passenger driven scenario performs well if there are several passengers waiting at a stop far ahead as passenger draw the vehicle towards the stop. On the other hand, the passenger driven strategy considers the adverse effect of bus bunching too. Therefore, it can happen, that the two objectives considered in this strategy (i.e. minimizing passenger wait and maintaining equidistant headways) are in conflict. Although it is possible for some stops that the strategy falls behind, in the long run, on network level it provides the least passenger wait. The average passenger waiting times for the bus at each stop are summarized in Table 4 and their second means are (mean of every departure and every stop) as follows: $177.63 \mathrm{~s}$ for HCS, $169.77 \mathrm{~s}$ for passenger driven, and $200.17 \mathrm{~s}$ for balanced control. 

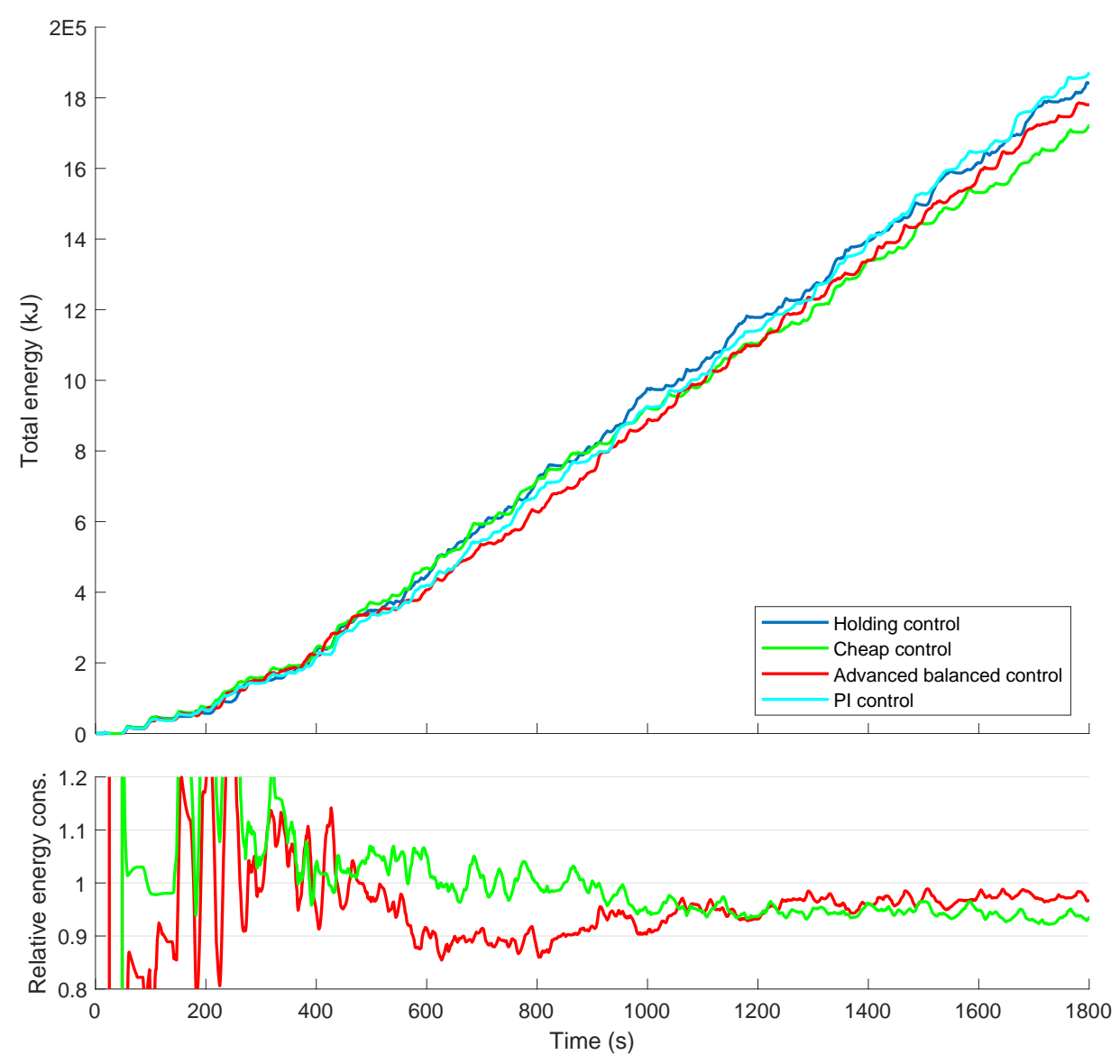

Figure 14: Total energy consumption on the bus line. The relative energy consumptions are normalized with the holding control strategy

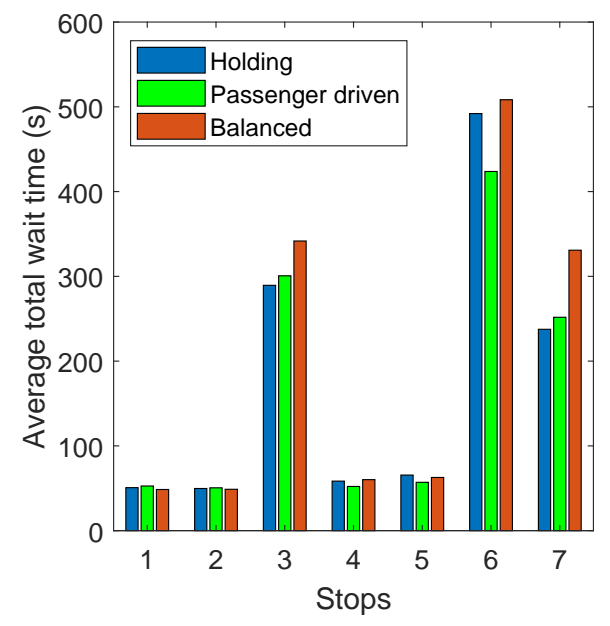

Table 4: Average total waiting time (in seconds) of passengers at each stop

\begin{tabular}{llll} 
Stop ID & $\begin{array}{l}\text { Holding } \\
\text { control }\end{array}$ & $\begin{array}{l}\text { Pass. } \\
\text { driven }\end{array}$ & $\begin{array}{l}\text { Adv. balanced } \\
\text { control }\end{array}$ \\
\hline Stop 1 & 50.7 & 52.6 & 48.5 \\
Stop 2 & 49.7 & 50.5 & 48.8 \\
Stop 3 & 289.4 & 300.6 & 341.7 \\
Stop 4 & 58.4 & 52.1 & 60.1 \\
Stop 5 & 65.5 & 57.0 & 62.7 \\
Stop 6 & 492.0 & 423.7 & 508.3 \\
Stop 7 & 237.5 & 251.7 & 330.8 \\
\hline
\end{tabular}

Figure 15: Average total waiting time of passengers at each stop 


\section{Conclusions and future work}

In the paper, multi-objective control strategies were presented combined into one optimization framework to enhance bus operation on an urban bus network. The proposed velocity control considers four conflicting goals: elimination of bunching and timetable reliability, as well as reducing energy consumption and passenger waiting times. To this end, three models were formulated. i) Based on a linear bus following model and three reference trajectories (leading bus, following bus and an idealized timetable-based trajectory) a a rolling horizon optimization problem was created. Using the trajectories of the leader and follower buses a headway tracking objective was formulated. In the same vein, based on an idealized bus trajectory, timetable tracking was taken into account. ii) To consider energy efficient operation of electric buses, a first principal energy consumption model was incorporated into the optimization. The model considers battery electric vehicles with regenerative braking and penalizes rapid accelerations/decelerations. iii) A passenger waiting model was introduced, modeling the accumulation of passengers at stops ahead. With the above models combined a distributed model predictive controller was formulated calculating an optimal velocity profile for one bus. Via different weighting of the considered objectives, six different control strategies were chosen and compared via a traffic simulation environment. As benchmark control strategies, HCS and PI controllers were selected. The analysis focused mainly on the passenger waiting and energy consumption objectives. The main findings of the numerical simulations are summarized below.

- Since the holding control only considers a static timetable, it performs similar to the multi-objective control strategies in terms of punctuality and headway adherence.

- On the other hand, in terms of energy consumption holding control strategy performs significantly worse than the proposed energy-aware solutions. The noticeable difference is that velocity profiles become smoother as buses approach stops. Using the HCS as a benchmark (100\%), the energy-aware strategy consumes $8 \%$ less, while the advanced balanced velocity control consumes $3 \%$ less on a network level. On network level, in long-run, significant energy savings can be achieved via the proposed velocity control.

- Finally, passenger waiting times were analyzed. In this metric HCS performs well, as it spends significantly more time at stops compared to other strategies. However, the passenger driven control solution outperforms it as it considers passengers waiting several stops ahead. Compared to the holding strategy, on average, the passenger driven control reduces the passenger waiting times by $4.6 \%$. The balanced control performs much worse in this metric (due to considering other objectives, such as energy consumption), the average passenger waiting time is increased by $11.2 \%$.

The proposed control framework provides a flexible selection of objectives, depending on the prevailing traffic situation. As a future research direction, the proposed strategies should be further analyzed in a wide variety of traffic scenarios. This would lead us to formulate adaptive weighting strategies, further enhancing the efficiency of the bus fleet control system. In addition, considering battery capacity and maintenance related constraints could be further investigated.

\section{Acknowledgements}

The research reported in this paper was supported by the János Bolyai Research Scholarship of the Hungarian Academy of Sciences. The authors acknowledge the contribution of Transport 
Area of Advance at Chalmers University of Technology. The project has been partially supported by Energimyndigheten through the project 'Operational Network Energy Management for Electrified buses' (46365-1).

\section{References}

[1] J. M. Pilachowski, An approach to reducing bus bunching, PhD Thesis, University of California, Berkeley, 2009.

[2] J. Ap. Sorratini, R. Liu, S. Sinha, Assessing bus transport reliability using microsimulation, Transportation Planning and Technology 31 (3) (2008) 303-324. doi:10.1080/ 03081060802086512

[3] A. Fonzone, J.-D. Schmöcker, R. Liu, A model of bus bunching under reliability-based passenger arrival patterns, Transportation Research Part C: Emerging Technologies 59 (2015) 164-182. doi:10.1016/j.trc.2015.05.020.

[4] C. F. Daganzo, A headway-based approach to eliminate bus bunching: Systematic analysis and comparisons, Transportation Research Part B: Methodological 43 (10) (2009) 913-921. doi:10.1016/j.trb.2009.04.002.

[5] C. F. Daganzo, J. Pilachowski, Reducing bunching with bus-to-bus cooperation, Transportation Research Part B: Methodological 45 (1) (2011) 267-277. doi:10.1016/j.trb. 2010.06 .005 .

[6] W. Wu, R. Liu, W. Jin, Modelling bus bunching and holding control with vehicle overtaking and distributed passenger boarding behaviour, Transportation Research Part B: Methodological 104 (2017) 175-197. doi:10.1016/j.trb.2017.06.019.

[7] K. Ampountolas, M. Kring, Mitigating bunching with bus-following models and bus-to-bus cooperation, in: IEEE 18th International Conference on Intelligent Transportation Systems (ITSC), 15 - 18 September, 2015, Las Palmas, Spain, pp. 60-65. doi:10.1109/ITSC.2015. 18 .

[8] J. J. Bartholdi, D. D. Eisenstein, A self-coördinating bus route to resist bus bunching, Transportation Research Part B: Methodological 46 (4) (2012) 481-491. doi:10.1016/j. trb.2011.11.001.

[9] M. M. Rahman, S. Wirasinghe, L. Kattan, Analysis of bus travel time distributions for varying horizons and real-time applications, Transportation Research Part C: Emerging Technologies 86 (2018) 453-466. doi:10.1016/j.trc.2017.11.023.

[10] M. Estrada, J. Mensión, J. M. Aymamí, L. Torres, Bus control strategies in corridors with signalized intersections, Transportation Research Part C: Emerging Technologies 71 (2016) 500-520. doi:10.1016/j.trc.2016.08.013.

[11] M. M. Nesheli, A. A. Ceder, T. Liu, A robust, tactic-based, real-time framework for publictransport transfer synchronization, Transportation Research Part C: Emerging Technologies 60 (2015) 105-123. doi:10.1016/j.trc.2015.08.008.

[12] M. Gallet, T. Massier, T. Hamacher, Estimation of the energy demand of electric buses based on real-world data for large-scale public transport networks, Applied energy 230 (2018) 344-356. doi:10.1016/j.apenergy.2018.08.086. 
[13] B. Németh, P. Gáspár, Road inclinations in the design of lpv-based adaptive cruise control, IFAC Proceedings Volumes 44 (1) (2011) 2202-2207. doi:10.3182/20110828-6-IT-1002. 00932

[14] B. Saerens, H. Rakha, M. Diehl, E. Van den Bulck, A methodology for assessing eco-cruise control for passenger vehicles, Transportation research part D: transport and environment 19 (2013) 20-27.

[15] S. Akhegaonkar, L. Nouveliere, S. Glaser, F. Holzmann, Smart and green ACC: energy and safety optimization strategies for EVs, IEEE Transactions on Systems, Man, and Cybernetics: Systems 48 (1) (2018) 142-153. doi:10.1109/TSMC.2016.2600273.

[16] A. Mihály, B. Németh, P. Gáspár, Real-time look-ahead cruise control simulator, Periodica Polytechnica Transportation Engineering 46 (1) (2018) 11-16. doi:10.3311/PPtr.9896.

[17] E. Kural, S. Jones, A. F. Parrilla, A. Grauers, Traffic light assistant system for optimized energy consumption in an electric vehicle, in: 2014 International Conference on Connected Vehicles and Expo (ICCVE), IEEE, 2014. doi:10.1109/iccve.2014.7297619.

[18] J. D. Vreeswijk, M. Mahmod, B. van Arem, Energy efficient traffic management and controlthe eCoMove approach and expected benefits, in: 13th International IEEE Conference on Intelligent Transportation Systems, IEEE, 19-22 September 2010, Funchal, Portugal, pp. 955-961. doi:10.1109/ITSC.2010.5625122.

[19] C. Lv, X. Hu, A. Sangiovanni-Vincentelli, Y. Li, C. M. Martinez, D. Cao, Driving-stylebased codesign optimization of an automated electric vehicle: A cyber-physical system approach, IEEE Transactions on Industrial Electronics 66 (4) (2019) 2965-2975. doi: 10.1109/tie.2018.2850031.

[20] Z. Bi, L. Song, R. De Kleine, C. C. Mi, G. A. Keoleian, Plug-in vs. wireless charging: Life cycle energy and greenhouse gas emissions for an electric bus system, Applied Energy 146 (2015) 11-19. doi:10.1016/j.apenergy.2015.02.031.

[21] M. Rogge, E. van der Hurk, A. Larsen, D. U. Sauer, Electric bus fleet size and mix problem with optimization of charging infrastructure, Applied Energy 211 (2018) 282-295. doi: 10.1016/j.apenergy.2017.11.051.

[22] M. Rupp, N. Handschuh, C. Rieke, I. Kuperjans, Contribution of country-specific electricity mix and charging time to environmental impact of battery electric vehicles: A case study of electric buses in germany, Applied Energy 237 (2019) 618-634. doi:10.1016/j.apenergy . 2019.01.059.

[23] M. H. Amini, A panorama of interdependent power systems and electrified transportation networks, in: Studies in Systems, Decision and Control, Springer International Publishing, 2018, pp. 23-41. doi:10.1007/978-3-319-98923-5_2.

[24] M. Rogge, S. Wollny, D. Sauer, Fast charging battery buses for the electrification of urban public transport - a feasibility study focusing on charging infrastructure and energy storage requirements, Energies 8 (5) (2015) 4587-4606. doi:10.3390/en8054587.

[25] W. Su, H. Eichi, W. Zeng, M.-Y. Chow, A survey on the electrification of transportation in a smart grid environment, IEEE Transactions on Industrial Informatics 8 (1) (2012) 1-10. doi:10.1109/tii.2011.2172454. 
[26] M. Mohamed, H. Farag, N. El-Taweel, M. Ferguson, Simulation of electric buses on a full transit network: Operational feasibility and grid impact analysis, Electric Power Systems Research 142 (2017) 163-175. doi:10.1016/j.epsr.2016.09.032.

[27] S. Yang, J. Wu, H. Sun, X. Yang, Z. Gao, A. Chen, Bi-objective nonlinear programming with minimum energy consumption and passenger waiting time for metro systems, based on the real-world smart-card data, Transportmetrica B: Transport Dynamics (2017) 118doi:10.1080/21680566.2017.1320775.

[28] Y. Xuan, J. Argote, C. F. Daganzo, Dynamic bus holding strategies for schedule reliability: Optimal linear control and performance analysis, Transportation Research Part B: Methodological 45 (10) (2011) 1831-1845. doi:10.1016/j.trb.2011.07.009.

[29] M. Andres, R. Nair, A predictive-control framework to address bus bunching, Transportation Research Part B: Methodological 104 (2017) 123-148. doi:10.1016/j.trb.2017.06. 013 .

[30] B. Varga, T. Tettamanti, B. Kulcsár, Optimally combined headway and timetable reliable public transport system, Transportation Research Part C: Emerging Technologies 92 (2018) 1-26. doi:10.1016/j.trc.2018.04.016

[31] T. Tettamanti, I. Varga, Z. Szalay, Impacts of autonomous cars from a traffic engineering perspective, Periodica Polytechnica Transportation Engineering 44 (4) (2016) 244-250. doi:10.3311/PPtr.9464.

[32] M. Bando, K. Hasebe, A. Nakayama, A. Shibata, Y. Sugiyamai, Dynamical model of traffic congestion and numerical simulation, Physical Review E 51 (2) (1995) 1035-1043. doi: 10.1103/PhysRevE.51.1035

[33] D. Helbing, B. Tilch, Generalized force model of traffic dynamics, Physical Review E 58 (133) (1998) 1-6. doi:10.1103/PhysRevE.58.133.

[34] A. Kesting, M. Treiber, M. Schönhof, D. Helbing, Adaptive cruise control design for active congestion avoidance, Transportation Research Part C: Emerging Technologies 16 (6) (2008) 668-683. doi:10.1016/j.trc.2007.12.004.

[35] S. P. Hoogendoorn, P. H. Bovy, State-of-the-art of vehicular traffic flow modelling, Proceedings of the Institution of Mechanical Engineers, Part I: Journal of Systems and Control Engineering 215 (4) (2001) 283-303.

[36] M. Van den Berg, A. Hegyi, B. De Schutter, J. Hellendoorn, A macroscopic traffic flow model for integrated control of freeway and urban traffic networks, in: Decision and Control, 2003. Proceedings. 42nd IEEE Conference on, Vol. 3, IEEE, 2003, pp. 2774-2779.

[37] J. M. Maciejowski, Predictive control: with constraints, Prentice Hall, Harlow, UK, 2002.

[38] L. Guzzella, A. Sciarretta, et al., Vehicle propulsion systems, Vol. 3, Springer-Verlag Berlin Heidelberg, 2013. doi:10.1007/978-3-642-35913-2.

[39] M. Barth, K. Boriboonsomsin, Energy and emissions impacts of a freeway-based dynamic eco-driving system, Transportation Research Part D: Transport and Environment 14 (6) (2009) 400-410. doi:10.1016/j.trd.2009.01.004. 
[40] O. Olsson, A. Grauers, S. Pettersson, Method to analyze cost effectiveness of different electric bus systems, in: EVS29 International Battery, Hybrid and Fuel Cell Electric Vehicle Symposium, 19-22 Montreal, Quebec, Canada, pp. 1-12.

[41] C. Lv, Y. Xing, C. Lu, Y. Liu, H. Guo, H. Gao, D. Cao, Hybrid-learning-based classification and quantitative inference of driver braking intensity of an electrified vehicle, IEEE Transactions on Vehicular Technology (2018) 1-1doi:10.1109/tvt.2018.2808359

[42] G. Garcia-Bunster, M. Torres-Torriti, A density-based approach for effective pedestrian counting at bus stops, in: IEEE International Conference on Systems Man and Cybernetics (SMC), 11-14 October 2009, San Antonio, TX, USA, pp. 3434-3439. doi:ICSMC.2009. 5346178 .

[43] M. Luethi, U. Weidmann, A. Nash, Passenger arrival rates at public transport stations, in: 86th Transportation Research Board Annual Meeting (TRB 2007), Institute for Transport Planning and Systems, 21-25 January 2007, Washington, D.C., USA, 2007, pp. 1-12.

[44] M. Dessouky, R. Hall, L. Zhang, A. Singh, Real-time control of buses for schedule coordination at a terminal, Transportation Research Part A: Policy and Practice 37 (2) (2003) 145-164. doi:10.1016/S0965-8564(02)00010-1.

[45] J. Nocedal, S. J. Wright, Numerical optimization, 2nd Edition, Springer-Verlag New York, 2006. doi:10.1007/978-0-387-40065-5.

[46] PTV, Stumpfstrasse 1, D-76131 Karlsruhe, Germany, VISSIM 7.0 User Manual (2014).

[47] S. Kullback, R. A. Leibler, On information and sufficiency, The Annals of Mathematical Statistics 22 (1) (1951) 79-86. doi:10.1214/aoms/1177729694.

[48] I. I. Sirmatel, N. Geroliminis, Hybrid model predictive control of public transport operations, in: 18th Swiss Transport Research Conference, May 16-18, 2018, Monte Verita, Ascona, Switzerland, 2018. 